\title{
Inexact accurate partitioned algorithms for fluid-structure interaction problems with finite elasticity in haemodynamics
}

\author{
Fabio Nobile ${ }^{a}$, Matteo Pozzoli ${ }^{b}$, Christian Vergara $^{\mathrm{c}, *}$ \\ a CSQI - MATHICSE, École Polytechnique Fédérale de Lausanne, Switzerland \\ ${ }^{\mathrm{b}}$ AgustaWestland - A Finmeccanica Company, Cascina Costa (VA), Italy \\ c Dipartimento di Ingegneria, Università degli Studi Bergamo, Italy
}

\section{A R T I C L E I N F O}

\section{Article history:}

Received 21 January 2014

Received in revised form 16 April 2014

Accepted 16 May 2014

Available online 22 May 2014

\section{Keywords:}

Fluid-structure interaction

Finite elasticity

Robin transmission conditions

BDF schemes

Newton method

Haemodynamics

\begin{abstract}
A B S T R A C T
In this paper we consider the numerical solution of the three-dimensional fluid-structure interaction problem in haemodynamics, in the case of real geometries, physiological data and finite elasticity vessel deformations. We study some new inexact schemes, obtained from semi-implicit approximations, which treat exactly the physical interface conditions while performing just one or few iterations for the management of the interface position and of the fluid and structure non-linearities. We show that such schemes allow to improve the efficiency while preserving the accuracy of the related exact (implicit) scheme. To do this we consider both a simple analytical test case and two real cases of clinical interest in haemodynamics. We also provide an error analysis for a simple differential model problem when a BDF method is considered for the time discretization and only few Newton iterations are performed at each temporal instant.
\end{abstract}

(c) 2014 Elsevier Inc. All rights reserved.

\section{Introduction}

To obtain predictive accurate information about the blood fluid-dynamics in the arteries, one needs to consider the interaction between the blood and the vascular vessel. This leads to the solution of a fluid-structure interaction (FSI) problem in three-dimensional (3D) real geometries [44,7,18,47,15,3,20,21]. To capture the complex dynamics characterizing such a problem, non-linear fluid and structure models have to be taken into account, leading to a complex non-linear coupled problem, formed also by the fluid domain subproblem when the fluid equations are written in Arbitrary Lagrangian-Eulerian (ALE) formulation $[29,14]$.

Efficient numerical strategies are mandatory to solve the FSI problem with non-linear fluid and structure models, in 3D real geometries and with physiological data. Only few works have focused on this aspect. We mention [7,25,37] among the monolithic schemes, which build the whole non-linear system, and [33] among the partitioned schemes, which consist in the successive solution of the subproblems in an iterative framework (see also $[15,5,11,4,10]$ in the case of the linear elasticity). In this work, we focus on partitioned strategies, whose main difficulties are:

1. The high added mass effect, due to the similar fluid and structure densities, which makes the solution of the FSI problem very difficult $[9,24,43]$;

\footnotetext{
* Corresponding author at: Viale Marconi 5, 24044 Dalmine (BG), Italy. Tel.: +39 0352052314; fax: +39 0352052310.

E-mail addresses: fabio.nobile@epfl.ch (F. Nobile), mattepozzoli@gmail.com (M. Pozzoli), christian.vergara@unibg.it (C. Vergara).
} 
2. The treatment of the physical interface conditions, which enforce the continuity of velocities and tractions at the fluidstructure (FS) interface between the fluid and the structure subproblems;

3. The treatment of the constitutive non-linearities in the fluid and structure models;

4. The treatment of the geometrical interface condition, which enforces the continuity of displacements at the FS interface between the fluid and the structure domains.

Regarding points 1 and 2, it has been clearly highlighted in several works that the physical conditions should be treated implicitly in haemodynamics, due to the high added mass effect $[9,24,3,43,51]$. However, some recent works proposed suitable explicit strategies which allow to obtain stable results. In particular, we mention [17], where the authors treated the case of a thin-walled structure by using Robin interface conditions, and [8], where the authors obtained stable results for the case of a thick structure by applying the Nitsche's method in combination with a suitable interface stabilization term. This new generation of schemes is very promising in view of the reduction of the computational cost. However, their accuracy is still an open issue for real haemodynamic applications. In this work we consider partitioned algorithms, which treats implicitly the physical conditions, based on Robin interface conditions, which have good convergence properties, independent of the added-mass effect $[3,4,1,26,41]$.

As for point 3, we have to consider the fluid and the structure constitutive non-linearities. A first approach to treat them consists in solving the non-linear fluid and structure subproblems in an iterative framework until convergence of the physical interface conditions (think for example to the classical Dirichlet-Neumann scheme $[32,34,28,30,43]$ ). Each iteration involves the solution of two non-linear subproblems, the fluid problem (for example through Picard iterations), and the structure problem (with the Newton method). In this case, the constitutive non-linearities are treated in an inner loop with respect to both the physical and the geometrical interface conditions. We refer to these schemes as "classical" partitioned algorithms. A second strategy considered so far consists in applying the Newton or the approximate-Newton method (the latter obtained by approximating the Jacobian) to the monolithic non-linear system (approximate-Newton-based algorithms). In [27], the author proposed a block-diagonal approximation of the Jacobian, leading to a partitioned algorithm where all the interface conditions and non-linearities are treated in the same loop (see also [36,28,33,13,48]). In [41], the authors considered alternative approximations of the Jacobian, leading to algorithms whose general structure consists in an external loop to manage the geometrical interface condition and the constitutive non-linearities and in an internal one to prescribe the physical interface conditions.

For what concerns point 4 , we can distinguish between exact and inexact strategies. The first group consists in those schemes that satisfy exactly the geometrical interface condition (geometrical exact schemes $[27,12,7,16])$. On the contrary, in the geometrical inexact schemes this condition is not satisfied, due to an explicit treatment of the interface position by extrapolation from previous time steps (the so-called semi-implicit schemes [15,6,42]), or to an a priori fixed number of fixed-point or Newton iterations performed over the interface position [41]. In the framework of the approximate-Newton methods such schemes could be obtained by performing just few iterations [41]. For example, the semi-implicit scheme can be thought as one iteration of the approximate-Newton method.

The geometrical inexact schemes have been shown to be stable and accurate in haemodynamics $[40,46,15,42,39,2,38,41]$. It is moreover well accepted that also an inexact treatment of the fluid non-linearity is enough to recover accurate and stable solutions $[15,6,41]$. All these inexact schemes have been studied in combination either with the linear elasticity or with an exact treatment of the structure non-linearity. Therefore, it is worth asking if in haemodynamic applications with finite elasticity one really needs to solve exactly the structure non-linearity, or if the latter can be linearized around a state suitably extrapolated from previous time steps together with the fluid convective term and the FS interface position. In this work, we propose new inexact schemes derived by considering just few approximate-Newton iterations over the monolithic system at each time step, leading to an inexact treatment of the geometrical coupling, of the fluid non-linearity and also of the structure non-linearity.

To study theoretically such inexact schemes, we provide for a simple model problem an error analysis when a BDF scheme is used in combination with one or few Newton or approximate-Newton iterations to solve the non-linear problem arising after the time discretization. Such analysis showed that the global accuracy depends on the order of the BDF scheme, the order of the extrapolation used to build the initial guess and the number of Newton or approximate-Newton iterations. To test the accuracy of the proposed schemes for FSI problems, we first considered a simple analytical test case. The numerical results confirmed the theoretical results found for the model problem. In particular, when a globally third order accurate time discretization of the FSI problem is considered, performing at least two approximate-Newton iterations allowed to recover a third order convergence in time even when starting from a first order extrapolation, while one approximate-Newton iteration was enough when starting form a third order extrapolation. Finally, we showed that the proposed inexact schemes are very accurate for real 3D cases when comparing the solution with that obtained with exact schemes, allowing to improve the computational efficiency up to three times.

The outline of the work is as follows. In Section 2 we present the global FSI problem, its time discretization and a Lagrange multipliers formulation useful to derive the numerical schemes. In Section 3 we present the new inexact schemes, and in Section 3.1 we provide an error analysis of inexact schemes for a model problem. In Section 4 we detail a particular choice of the internal tolerance for the exact case. Finally, in Section 5 we show several numerical results. In particular, in Section 5.1 we study the efficiency of exact schemes by considering two real cases in haemodynamics, in Section 5.2 we 

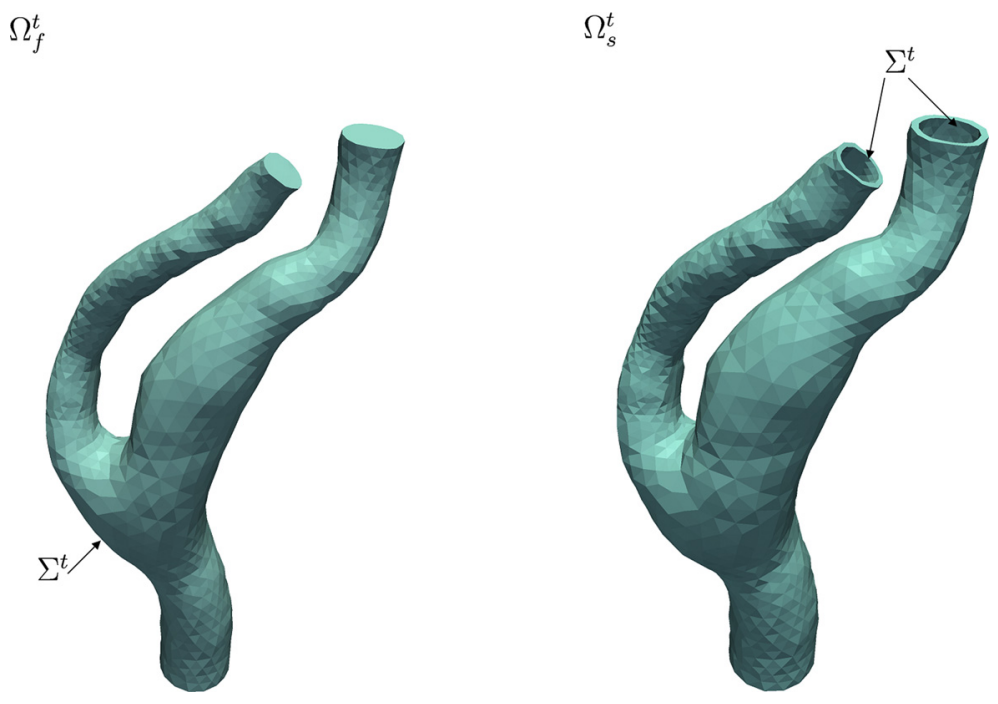

Fig. 1. Representation of the domain of the FSI problem: fluid domain on the left, structure domain on the right.

show the convergence rates of inexact schemes for an analytical test case, and in Section 5.3 we provide a study on the accuracy and efficiency of inexact schemes for real haemodynamic cases.

\section{The FSI problem and its time discretization}

Referring to the fluid domain $\Omega_{f}^{t}$ like the one represented in Fig. 1, left, we denote, for any function $v$ living in the current fluid configuration, by $\widetilde{v}:=v \circ \mathcal{A}$ its counterpart in the reference configuration $\Omega_{f}^{0}$, where $\mathcal{A}$ is the ALE map. By considering, instead, the structure domain $\Omega_{s}^{t}$ like the one represented in Fig. 1, right, we denote, for any function $g$ defined in the current solid configuration, by $\widetilde{g}:=g \circ \mathcal{L}$ its counterpart in the reference domain $\Omega_{s}^{0}$, where $\mathcal{L}$ is the Lagrangian map. The strong formulation of the FSI problem, including the computation of the ALE map reads then as follows:

Find, at each time $t \in(0, T]$, fluid velocity $\boldsymbol{u}_{f}$, pressure $p_{f}$, structure displacement $\boldsymbol{\eta}_{s}$ and fluid domain displacement $\boldsymbol{\eta}_{m}$, such that

$$
\begin{cases}-\Delta \widetilde{\boldsymbol{\eta}}_{m}=\mathbf{0} & \text { on } \Omega_{f}^{0}, \\ \widetilde{\boldsymbol{\eta}}_{m}=\widetilde{\boldsymbol{\eta}}_{s} & \text { on } \Sigma^{0}, \\ \rho_{f} \frac{D^{A} \boldsymbol{u}_{f}}{D t}+\rho_{f}\left(\left(\boldsymbol{u}_{f}-\boldsymbol{u}_{m}\right) \cdot \nabla\right) \boldsymbol{u}_{f}-\nabla \cdot \boldsymbol{T}_{f}\left(\boldsymbol{u}_{f}, p_{f}\right)=\boldsymbol{f}_{f} & \text { in } \Omega_{f}^{t}, \\ \nabla \cdot \boldsymbol{u}_{f}=0 & \text { in } \Omega_{f}^{t}, \\ \boldsymbol{u}_{f}=\frac{\partial \boldsymbol{\eta}_{s}}{\partial t} & \text { on } \Sigma^{t}, \\ \boldsymbol{T}_{s}\left(\boldsymbol{\eta}_{s}\right) \boldsymbol{n}-\boldsymbol{T}_{f}\left(\boldsymbol{u}_{f}, p_{f}\right) \boldsymbol{n}=\mathbf{0} & \text { on } \Sigma^{t}, \\ \rho_{s} \frac{\partial^{2} \widetilde{\boldsymbol{\eta}}_{s}}{\partial t^{2}}-\nabla \cdot \widetilde{\boldsymbol{T}}_{s}\left(\widetilde{\boldsymbol{\eta}}_{s}\right)=\widetilde{\boldsymbol{f}}_{s} & \text { in } \Omega_{s}^{0},\end{cases}
$$

and then find accordingly the fluid domain velocity $\widetilde{\boldsymbol{u}}_{m}:=\frac{\partial \widetilde{\boldsymbol{\eta}}_{m}}{\partial t}$, the ALE map and the new points $\boldsymbol{x}_{f}^{t}$ of the fluid domain by moving the points $\boldsymbol{x}_{f}^{0}$ of the reference domain $\Omega_{f}^{0}$ :

$$
\mathcal{A}\left(\boldsymbol{x}_{f}^{0}\right)=\boldsymbol{x}_{f}^{t}=\boldsymbol{x}_{f}^{0}+\widetilde{\boldsymbol{\eta}}_{m} .
$$

In the previous problem $\rho_{f}$ and $\rho_{s}$ are the fluid and structure densities, $\mu$ is the constant blood viscosity, $\boldsymbol{f}_{f}$ and $\boldsymbol{f}_{s}$ the forcing terms, $\boldsymbol{n}$ the unit normal exiting from the structure domain, $\frac{D^{A}}{D t}$ denotes the ALE derivative, $\boldsymbol{T}_{f}\left(\boldsymbol{u}_{f}, p_{f}\right)$ is the Cauchy stress tensor related to a homogeneous, Newtonian, incompressible fluid, whereas $\widetilde{\boldsymbol{T}}_{s}\left(\widetilde{\boldsymbol{\eta}}_{s}\right)$ and $\boldsymbol{T}_{s}\left(\boldsymbol{\eta}_{s}\right)$ are the first Piola-Kirchhoff and the Cauchy stress tensors of the solid, respectively, describing the structure problem. The two matching conditions enforced at the FS interface are the continuity of velocities (1) 5 and the continuity of tractions (1)6 (physical interface conditions), whilst condition $(1)_{2}$ enforces the continuity at the FS interface of displacements of the 
fluid and structure subdomains (geometrical interface condition). Problem (1) has to be endowed with suitable boundary conditions on $\Omega_{f}^{t} \backslash \Sigma^{t}$ and $\Omega_{s}^{0} \backslash \Sigma^{0}$, and with suitable initial conditions.

Let $\Delta t$ be the time discretization parameter and $t^{n}:=n \Delta t, n=0,1, \ldots$ For a generic function $z$, we denote with $z^{n}$ the approximation of $z\left(t^{n}\right)$. We consider Backward Differentiation Formulae of order $p$ (BDFp) of the form

$$
\begin{aligned}
\frac{D_{p} v^{n+1}}{\Delta t}:=\frac{1}{\Delta t}\left(\beta_{0} v^{n+1}-\sum_{i=1}^{p} \beta_{i} v^{n+1-i}\right)=\frac{\partial v}{\partial t}\left(t^{n+1}\right)+O\left(\Delta t^{p}\right), \\
\frac{D_{p}^{2} v^{n+1}}{\Delta t^{2}}:=\frac{1}{\Delta t^{2}}\left(\xi_{0} v^{n+1}-\sum_{i=1}^{p+1} \xi_{i} v^{n+1-i}\right)=\frac{\partial^{2} v}{\partial t^{2}}\left(t^{n+1}\right)+O\left(\Delta t^{p}\right),
\end{aligned}
$$

for suitable coefficients $\beta_{i}$ and $\xi_{i}[41,43]$. We report here the formulation of the time discretization of order $p$ of problem (1).

Given the solution at previous time steps, find the fluid velocity $\boldsymbol{u}_{f}^{n+1}$, the pressure $p_{f}^{n+1}$, the structure displacement $\eta_{s}^{n+1}$ and the fluid domain displacement $\eta_{m}^{n+1}$, such that

$$
\left\{\begin{array}{lc}
-\Delta \widetilde{\boldsymbol{\eta}}_{m}^{n+1}=\mathbf{0} & \text { in } \Omega_{f}^{0}, \\
\widetilde{\boldsymbol{\eta}}_{m}^{n+1}=\widetilde{\boldsymbol{\eta}}_{s}^{n+1} & \text { on } \Sigma^{0}, \\
\rho_{f} \frac{\boldsymbol{u}_{f}^{n+1}}{\Delta t}+\rho_{f}\left(\left(\boldsymbol{u}_{f}^{n+1}-\boldsymbol{u}_{m}^{n+1}\right) \cdot \nabla\right) \boldsymbol{u}_{f}^{n+1}-\nabla \cdot \boldsymbol{T}_{f}\left(\boldsymbol{u}_{f}^{n+1}, p_{f}^{n+1}\right)=\boldsymbol{f}_{f}^{n+1} & \text { in } \Omega_{f}^{n+1}, \\
\nabla \cdot \boldsymbol{u}_{f}^{n+1}=0 & \text { in } \Omega_{f}^{n+1}, \\
\boldsymbol{u}_{f}^{n+1}=\boldsymbol{u}_{s}^{n+1} & \text { on } \Sigma^{n+1}, \\
\boldsymbol{T}_{s}\left(\boldsymbol{\eta}_{s}^{n+1}\right) \boldsymbol{n}-\boldsymbol{T}_{f}\left(\boldsymbol{u}_{f}^{n+1}, p_{f}^{n+1}\right) \boldsymbol{n}=\mathbf{0} & \text { on } \Sigma^{n+1}, \\
D_{p}^{2} \widetilde{\boldsymbol{\eta}}_{s}^{n+1}-\nabla \cdot \widetilde{\boldsymbol{T}}_{s}\left(\widetilde{\boldsymbol{\eta}}_{s}^{n+1}\right)=\widetilde{\boldsymbol{f}}_{s}^{n+1} & \text { in } \Omega_{s}^{0}, \\
\rho_{s} &
\end{array}\right.
$$

and then find accordingly the discrete fluid domain velocity $\widetilde{\boldsymbol{u}}_{m}^{n+1}$ and the points $\boldsymbol{x}_{f}^{n+1}$ of the new fluid domain by

$$
\widetilde{\boldsymbol{u}}_{m}^{n+1}:=\frac{D_{p} \widetilde{\boldsymbol{\eta}}_{m}^{n+1}}{\Delta t}, \quad \boldsymbol{x}_{f}^{n+1}=\boldsymbol{x}_{f}^{0}+\widetilde{\boldsymbol{\eta}}_{m}^{n+1} .
$$

Notice that in problem (3) we have also introduced the structure velocity $\boldsymbol{u}_{s}^{n}:=\frac{D_{p} \boldsymbol{\eta}_{s}^{n}}{\Delta t}$.

We consider here an equivalent formulation of (3) based on the introduction of three Lagrange multipliers living at the FS interface, representing the fluid and structure tractions, $\lambda_{f}$ and $\lambda_{s}$, and the normal derivative of the fluid mesh displacement, $\lambda_{m}$ [41]. For the sake of notation we remove the temporal index ${ }^{n+1}$. With $\Sigma_{f}^{D}, \Sigma_{s}^{D, 0}$ and $\Sigma_{m}^{D}$ we denote the parts of the boundary where Dirichlet boundary conditions are prescribed. Then, we define the following spaces

$$
\begin{array}{ll}
V_{f}:=\left\{v \in H^{1}\left(\Omega_{f}\right):\left.v\right|_{\Sigma_{f}^{D}}=0\right\}, & Q:=L^{2}\left(\Omega_{f}\right), \\
V_{s}:=\left\{v \in H^{1}\left(\Omega_{s}^{0}\right):\left.v\right|_{\Sigma_{s}^{D, 0}}=0\right\}, & V_{m}:=\left\{v \in H^{1}\left(\Omega_{f}^{0}\right):\left.v\right|_{\Sigma_{m}^{D, 0}}=0\right\} .
\end{array}
$$

Let $\boldsymbol{v}_{f}:=\left(\boldsymbol{u}_{f}, p_{f}\right)$ collect the fluid unknowns and $\mathcal{F}: \boldsymbol{V}_{f} \times Q \times \boldsymbol{V}_{m} \rightarrow\left(\boldsymbol{V}_{f} \times Q\right)^{\prime}$ be the fluid operator. Analogously, for the structure subproblem we define the operator $\mathcal{S}: \boldsymbol{V}_{S} \rightarrow\left(\boldsymbol{V}_{S}\right)^{\prime}$, and for the harmonic extension we introduce the operator $\mathcal{H}: \boldsymbol{V}_{m} \rightarrow\left(\boldsymbol{V}_{m}\right)^{\prime}$. We then rewrite problem (3) as follows

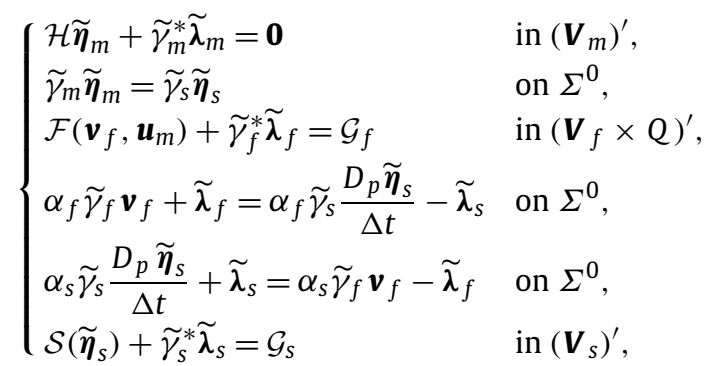

where $\tilde{\gamma}_{f}: \boldsymbol{V}_{f} \times Q \rightarrow \boldsymbol{H}^{1 / 2}\left(\Sigma^{0}\right), \tilde{\gamma}_{s}: \boldsymbol{V}_{s} \rightarrow \boldsymbol{H}^{1 / 2}\left(\Sigma^{0}\right), \tilde{\gamma}_{m}: \boldsymbol{V}_{m} \rightarrow \boldsymbol{H}^{1 / 2}\left(\Sigma^{0}\right)$ are trace operators and $\tilde{\gamma}_{f}^{*}, \tilde{\gamma}_{s}^{*}, \tilde{\gamma}_{m}^{*}$ are their adjoints, $\mathcal{G}_{s}$ and $\mathcal{G}_{f}$ account for the right hand sides, $(5)_{2}$ is the geometrical interface condition, and the physical interface 
conditions (5) $4-5$ are linear combinations of conditions (3) $)_{5-6}$ through coefficients $\alpha_{f}$ and $\alpha_{s}$. This will allow to obtain partitioned algorithms based on Robin interface conditions, which have good convergence properties, independent of the added-mass effect when the parameters $\alpha_{f}$ and $\alpha_{s}$ are suitably chosen $[3,4,1,26]$.

Observe that, since we solve the FSI problem in a partitioned way with Robin conditions at the FS interface (see (5)), the pressure is always defined and $L^{2}\left(\Omega_{f}\right)$ is the suitable pressure space for the weak formulation.

\subsection{Outlook of the iterative algorithms}

As discussed above, we have to face three sources of coupling and non-linearities, namely

(G) the geometrical interface condition;

(C) the constitutive fluid and structure non-linearities;

(P) the physical interface conditions.

We give here an outlook of the partitioned algorithms considered in the following sections. In principle, our model algorithm will consist of three nested loops, one for each of the three sources of coupling and non-linearities summarized above. Just to fix the ideas, we suppose here that the external loop will manage the geometrical interface condition, the intermediate one the constitutive non-linearities and the internal one the physical interface conditions. Our model algorithm is then of the type

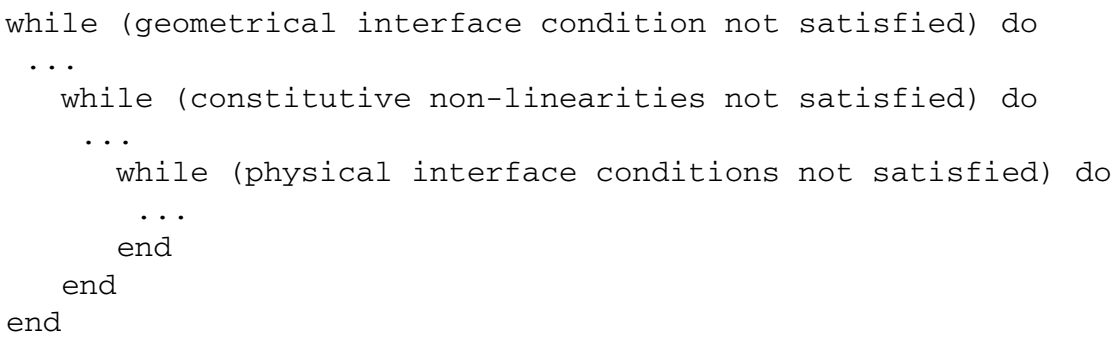

We call this algorithm $\mathrm{G} \infty-\mathrm{C} \infty-\mathrm{P} \infty$, where $\infty$ means that we let the iterations continue until convergence. Starting from this model scheme, we can obtain many other algorithms as follows.

1. The order of the loops could be exchanged, leading to different schemes $(\mathrm{G} \infty-\mathrm{P} \infty-\mathrm{C} \infty, \ldots)$;

2. We can merge two or more loops. For example, starting from the model algorithm $\mathrm{G} \infty-\mathrm{C} \infty$-P $\infty$, we could decide to treat in the same loop the geometrical interface condition and the constitutive non-linearities, obtaining the algorithm $\mathrm{GC} \infty-\mathrm{P} \infty$.

3. The external loop could be solved not until convergence, but performing just few external iterations. In this case, the corresponding algorithm is inexact, since the external stopping criterion is not satisfied. For example, starting from our model scheme, we could decide to perform only 2 external iterations, obtaining the algorithm I-G2-C $\infty-\mathrm{P} \infty$, where we put a letter $I$ at the beginning to emphasize that such scheme is inexact.

4. In the case of inexact schemes where only few (even one) iterations are performed in the external loop, we could consider starting the iterations from an initial guess obtained by a $q$-th order extrapolation in time. In this case, we add the letter $\mathrm{E}$ after the number of iterations of the loop involving the extrapolation. For example, starting from the model algorithm with just 1 external iterations, if we decided to use an extrapolation of the interface position as initial guess, we name the corresponding scheme as I-G1E-C $\infty-\mathrm{P} \infty$. In this work, we have always used the same order $p$ for the extrapolation as the order of the temporal scheme.

\section{Inexact schemes}

As discussed in the Introduction, in this work we want to extend the semi-implicit schemes $[46,15,6,42,10]$ and, more generally, the geometrical inexact schemes [41] to the case of finite elasticity structure models. In particular, we want to introduce a family of inexact schemes such that, together with the geometrical interface condition and the fluid constitutive non-linearity, also the structure constitutive non-linearity is not treated exactly. In other words, we ask if it is necessary in haemodynamic applications to handle exactly the structure constitutive non-linearity.

To do this, our starting point is the GC $\infty-\mathrm{P} \infty$ scheme introduced in [41]. Such an algorithm is obtained by applying the approximate-Newton method to the monolithic non-linear system (5), and by considering as approximation of the exact Jacobian 


$\left[\begin{array}{cc|cc|c}\mathcal{H} & \tilde{\gamma}_{m}^{*} & & & \\ \tilde{\gamma}_{m} & & & \tilde{\gamma}_{s}^{*} \\ \hline \nabla_{u_{m}} \mathcal{F} & \nabla_{v_{f}} \mathcal{F} & \tilde{\gamma}_{f}^{*} & & \\ & \alpha_{f} \tilde{\gamma}_{f} & I & I & -\alpha_{f} \frac{\beta_{s, 0}}{\Delta t} \widetilde{\gamma}_{s} \\ \hline & -\alpha_{s} \widetilde{\gamma}_{f} & I & I & \alpha_{s} \frac{\beta_{s, 0}}{\Delta t} \widetilde{\gamma}_{s} \\ & & & \tilde{\gamma}_{s}^{*} & \nabla_{\eta_{s}} \mathcal{S}\end{array}\right]$,

the following expression

$$
\widehat{J}_{D L}=\left[\begin{array}{cc|cc|c}
\mathcal{H} & \tilde{\gamma}_{m}^{*} & & & \\
\widetilde{\gamma}_{m} & & & \\
\hline & \widehat{\nabla}_{v_{f}} \mathcal{F} & \tilde{\gamma}_{f}^{*} & & \\
& \alpha_{f} \tilde{\gamma}_{f} & I & I & -\alpha_{f} \frac{\beta_{s, 0}}{\Delta t} \widetilde{\gamma}_{s} \\
\hline & -\alpha_{s} \widetilde{\gamma}_{f} & I & I & \alpha_{s} \frac{\beta_{s, 0}}{\Delta t} \widetilde{\gamma}_{s} \\
& & & \tilde{\gamma}_{s}^{*} & \nabla_{\eta_{s}} \mathcal{S}
\end{array}\right],
$$

which neglects the term $\nabla_{u_{m}} \mathcal{F}$ involving the shape derivatives and the term $\widetilde{\gamma}_{s}^{*}$ which couples the fluid geometry and the structure problems, and where $\widehat{\nabla}_{v_{f}} \mathcal{F}$ is the Oseen approximation of $\nabla_{v_{f}} \mathcal{F}$ [41]. This algorithm has a double-loop nature, where the physical interface conditions are managed in the internal loop.

Now, to derive the inexact schemes, we start from the observation that, in the case of the linear infinitesimal elasticity, the semi-implicit scheme, that treats explicitly the interface position, can be regarded as the GC $\infty-\mathrm{P} \infty$ scheme where the number of external/approximate-Newton iterations is fixed and equal to 1 [41]. Now, the idea is to perform one external/approximate-Newton iteration also in the case of an FSI system with a non-linear structure. We name this new algorithm I-GC1-P $\infty$. Of course, since in this scheme the constitutive non-linearities are treated in the external loop, we obtain an algorithm which treats inexactly the structure non-linearity. More generally, it is possible to perform $m$ external/approximate-Newton iterations for a fixed $m \geq 1$, obtaining the I-GCm-P $\infty$ scheme, detailed in Algorithm 1 .

\section{Algorithm $1 \mathrm{I}-\mathrm{GCm}-\mathrm{P} \infty$ scheme.}

Given the solutions $\widetilde{\boldsymbol{\eta}}_{s}^{k}$ and $\boldsymbol{u}_{f}^{k}, m \in \mathbb{N}^{+}$and $0<\omega \leq 1$

UNTIL $0 \leq k<m$ DO

1. Compute $\widetilde{\boldsymbol{\eta}}_{m}^{k+1}$ and $\widetilde{\lambda}_{m}^{k+1}$ by solving the harmonic extension for the fluid domain

$$
\begin{cases}\mathcal{H} \widetilde{\boldsymbol{\eta}}_{m}^{k+1}+\widetilde{\gamma}_{m}^{*} \widetilde{\lambda}_{m}^{k+1}=0 & \text { in }\left(\boldsymbol{V}_{m}\right)^{\prime}, \\ \tilde{\gamma}_{m} \widetilde{\boldsymbol{\eta}}_{m}^{k+1}=\widetilde{\gamma}_{s} \widetilde{\boldsymbol{\eta}}_{s}^{k} & \text { on } \Sigma^{0},\end{cases}
$$

obtaining the new fluid domain $\Omega_{f}^{k+1}$ and the fluid domain velocity $\boldsymbol{u}_{m}^{k+1}$ by using (4);

2. Solve the linearized FSI problem. For its solution, we consider the following partitioned algorithm:

Given the solutions $\eta_{s, l-1}^{k+1}$ and $\lambda_{s, l-1}^{k+1}$

UNTIL P convergence Do

(a) Compute $\boldsymbol{v}_{f, l}^{k+1}$ and $\tilde{\lambda}_{f, l}^{k+1}$ by solving the linearized fluid subproblem with a Robin condition at the FS interface

$$
\begin{cases}\widehat{\nabla}_{v_{f}} \mathcal{F}\left(\boldsymbol{u}_{f}^{k}-\boldsymbol{u}_{m}^{k+1}\right) \boldsymbol{v}_{f, l}^{k+1}+\tilde{\gamma}_{f}^{*} \widetilde{\lambda}_{f, l}^{k+1}=\mathcal{G}_{f} & \text { in }\left(\boldsymbol{V}_{f}\left(\boldsymbol{\eta}_{m}^{k+1}\right) \times Q_{f}\left(\boldsymbol{\eta}_{m}^{k+1}\right)\right)^{\prime}, \\ \alpha_{f} \gamma_{f} \boldsymbol{v}_{f, l}^{k+1}+\lambda_{f, l}^{k+1}=\alpha_{f} \gamma_{s} \frac{D_{p} \boldsymbol{\eta}_{s, l-1}^{k+1}}{\Delta t}-\lambda_{s, l-1}^{k+1} & \text { on } \Sigma^{k+1} ;\end{cases}
$$

(b) Compute $\widetilde{\boldsymbol{\eta}}_{s, l}^{k+1}$ and $\tilde{\lambda}_{s, l}^{k+1}$ by solving the linearized structure subproblem with a Robin condition at the FS interface

$$
\begin{cases}\nabla_{\eta} \mathcal{S}\left(\widetilde{\boldsymbol{\eta}}_{s}^{k}\right) \delta \widetilde{\boldsymbol{\eta}}_{s, l}^{k+1}+\widetilde{\gamma}_{s}^{*} \delta \widetilde{\lambda}_{s, l}^{k+1}=\mathcal{G}_{s}-\mathcal{S}\left(\widetilde{\boldsymbol{\eta}}_{s}^{k}\right)-\tilde{\gamma}_{s}^{*} \widetilde{\lambda}_{s}^{k} & \text { in }\left(\boldsymbol{V}_{s}\right)^{\prime}, \\ \alpha_{s} \widetilde{\gamma}_{s} \frac{D_{p} \widetilde{\boldsymbol{\eta}}_{s, l}^{k+1}}{\Delta t}-\widetilde{\lambda}_{s, l}^{k+1}=\alpha_{s} \tilde{\gamma}_{f} \widetilde{\boldsymbol{v}}_{f, l}^{k+1}-\widetilde{\lambda}_{f, l}^{k+1} & \text { on } \Sigma^{0}\end{cases}
$$

(c) Relaxation step

$$
\tilde{\boldsymbol{\eta}}_{s, l}^{k+1}=\omega \widetilde{\boldsymbol{\eta}}_{s, l}^{k+1}+(1-\omega) \widetilde{\boldsymbol{\eta}}_{s, l-1}^{k+1}, \quad \tilde{\lambda}_{s, l}^{k+1}=\omega \widetilde{\lambda}_{s, l}^{k+1}+(1-\omega) \tilde{\lambda}_{s, l-1}^{k+1}
$$

(d) Update: $l \rightarrow l+1$;

$$
\text { END DO }
$$

3. Update: $k \rightarrow k+1$. 
We observe that at, step 2a, we have indicated in brackets the fluid convective term and we have highlighted the dependence of $\boldsymbol{V}_{f}$ and $Q_{f}$ on $\boldsymbol{\eta}_{m}^{k+1}$ through the fluid domain updating. For the internal loop, we monitor the residual of condition $(7)_{2}$, whereas the stopping criteria on the geometric interface condition and on the fluid and structure constitutive non-linearities are not checked. Then, for Algorithm 1 the only source of coupling/non-linearity which is solved exactly are the physical interface conditions. This fact makes very interesting the study of the accuracy of such schemes, since there is no a priori evidence that the structure problem needs to be solved exactly in order to recover a global accurate solution in real applications in haemodynamics.

\subsection{Analysis of a model problem}

In this section we provide a temporal error analysis of a BDFp method used in combination with the Newton or with the approximate-Newton method to solve the non-linear problem arising after the time discretization, where however only $J$ iterations of such methods are performed. We refer in this section to such "inexact" methods as Newton- $J$ and approximate-Newton-J, respectively. Our model problem is the simple first order ordinary differential equation

$$
\left\{\begin{array}{l}
y^{\prime}(t)=f(y(t)), \quad t \in(0, T] \\
y(0)=y_{0}
\end{array}\right.
$$

where $f \in C^{2}$. By applying the BDFp method (see (2)) to the previous equation, we obtain

$$
\frac{\beta_{0} y^{n+1}-\sum_{i=1}^{p} \beta_{i} y^{n+1-i}}{\Delta t}=f\left(y^{n+1}\right)
$$

leading to the solution of the non-linear scalar equation $g\left(y^{n+1}\right)=0$, with

$$
g(x)=x-\frac{\Delta t}{\beta_{0}} f(x)-\sum_{i=1}^{p} \frac{\beta_{i}}{\beta_{0}} y^{n+1-i} .
$$

Now, we apply the Newton-J method or the approximate-Newton-J method to solve such an equation. We denote by $y_{J}^{n+1}$ the solution of such methods. In both cases we consider an extrapolation $E_{q}$ of order $q$ as initial guess at each time step $t^{n+1}$. More precisely, we set

$$
y_{0}^{n+1}=E_{q}\left(\boldsymbol{y}_{J}^{n, q}\right):=\sum_{i=1}^{q}(-1)^{i+1}\left(\begin{array}{l}
q \\
i
\end{array}\right) y_{J}^{n+1-i},
$$

where $\boldsymbol{y}_{J}^{n, q}=\left\{y_{J}^{n+1-q}, \ldots, y_{J}^{n}\right\}$. The global temporal error is then given by

$$
E=E(p, J, q)=\max _{n=1, \ldots, N}\left|y\left(t^{n}\right)-y_{J}^{n}\right|,
$$

where $N=T / \Delta t$ is the total number of time steps. To compute such an error, we write

$$
E \leq \max _{n=1, \ldots, N}\left|y\left(t^{n}\right)-y^{n}\right|+\max _{n=1, \ldots, N}\left|E_{J}^{n}\right|
$$

where $E_{J}^{n}:=\left|y^{n}-y_{J}^{n}\right|$. The first term is related to the error due to the time discretization, that is the error obtained by reaching convergence at each time step in the Newton or in the approximate-Newton methods (the solution being the same at convergence). In particular, for a BDFp scheme, we have $\max _{n=1, \ldots, N}\left|y\left(t^{n}\right)-y^{n}\right| \leq C_{0} \Delta t^{p}$, for a suitable constant $C_{0}$, provided that the exact solution $y(t)$ is smooth enough. Then, to compute the global temporal order of the Newton-J and of the approximate-Newton- $J$ methods, we have to provide an estimate for the term $E_{J}:=\max _{n=1, \ldots, N}\left|E_{J}^{n}\right|$, that is the error introduced by the fact that we are not reaching convergence in the Newton methods. This is what we are going to do in what follows.

We first recall the following result, which will be useful to our aims, see, e.g., [45].

Proposition 1. Consider the following discrete problem for $n \geq p-1$

$$
\beta_{0} z^{n+1}-\sum_{i=1}^{p} \beta_{i} z^{n+1-i}=\Delta \operatorname{tg}_{1}\left(z^{n+1}\right)+\Delta \operatorname{tg}_{2}\left(t^{n+1}\right),
$$

where the $\beta_{j}$ 's are the coefficient of the BDFp scheme, $g_{1}$ is a Lipschitz function of the unknown with Lipschitz constant L, and $g_{2}$ a function of time. Then, it follows

$$
\left|z^{n}\right| \leq K\left((p+1) \max _{j=0, \ldots, p-1}\left|z^{j}\right|+T \max _{j=p, \ldots, n}\left|g_{2}\left(t^{j}\right)\right|\right)
$$

where $z^{j}, j=0, \ldots, p-1$, are the initial conditions, and $K=2 K_{0} e^{2 T(p+2) L K_{0} \max _{i}\left|\beta_{i}\right|}$, with $K_{0}$ the stability constant of the problem with $g_{1}=0$. 
We start from the analysis of the Newton-J method. We have the following

Theorem 1. Suppose that $f_{y}$ and $f_{y y}$ are globally bounded in $\mathbb{R}$, i.e. there exist $L_{1}, L_{2}$ such that

$$
\left|f_{y}(z)\right| \leq L_{1}, \quad\left|f_{y y}(z)\right| \leq L_{2}
$$

for all $z$. Moreover, assume that $f_{y y}$ is continuous. Then, assuming that the solution $y^{n}$ exists for all $n$, there exists $\Delta t_{0}$ with

$$
\Delta t_{0}<\frac{\beta_{0}}{L_{1}}
$$

such that the BDFp method applied to (10) used in combination with the Newton-J method to solve equation (12) and with an extrapolation of order $q \leq p$ as initial guess at each time step, is globally of order

$$
r=\min \left\{p ; 2^{J}(1+q)-2\right\},
$$

for $\Delta t \leq \Delta t_{0}$.

Proof. The exact Newton method applied to Eq. (12) reads

$$
y_{k+1}^{n+1}=y_{k}^{n+1}-\frac{\beta_{0} y_{k}^{n+1}-\Delta t f\left(y_{k}^{n+1}\right)-\sum_{i=1}^{p} \beta_{i} y^{n+1-i}}{\beta_{0}-\Delta t f_{y}\left(y_{k}^{n+1}\right)},
$$

which is well defined thanks to hypothesis (15). This leads to

$$
y_{k+1}^{n+1}=\frac{\Delta t}{\beta_{0}}\left[f\left(y_{k}^{n+1}\right)+f_{y}\left(y_{k}^{n+1}\right)\left(y_{k+1}^{n+1}-y_{k}^{n+1}\right)\right]+\sum_{i=1}^{p} \frac{\beta_{i}}{\beta_{0}} y^{n+1-i} .
$$

If we suppose to have performed at the previous time steps only $J$ iterations, (17) becomes

$$
y_{k+1}^{n+1}=\frac{\Delta t}{\beta_{0}}\left[f\left(y_{k}^{n+1}\right)+f_{y}\left(y_{k}^{n+1}\right)\left(y_{k+1}^{n+1}-y_{k}^{n+1}\right)\right]+\sum_{i=1}^{p} \frac{\beta_{i}}{\beta_{0}} y_{J}^{n+1-i}
$$

Moreover, by writing the Taylor expansion around $y_{k}^{n+1}$, we obtain

$$
f\left(y^{n+1}\right)=f\left(y_{k}^{n+1}\right)+f_{y}\left(y_{k}^{n+1}\right)\left(y^{n+1}-y_{k}^{n+1}\right)+\frac{1}{2} f_{y y}\left(\xi_{k}^{n+1}\right)\left(y^{n+1}-y_{k}^{n+1}\right)^{2},
$$

for a suitable $\xi_{k}^{n+1} \in\left(\min \left\{y^{n+1} ; y_{k}^{n+1}\right\}, \max \left\{y^{n+1} ; y_{k}^{n+1}\right\}\right)$. Substituting the expression of $f\left(y^{n+1}\right)$ from (11) in the previous identity, we have

$$
y^{n+1}=\frac{\Delta t}{\beta_{0}}\left[f\left(y_{k}^{n+1}\right)+f_{y}\left(y_{k}^{n+1}\right)\left(y^{n+1}-y_{k}^{n+1}\right)+\frac{1}{2} f_{y y}\left(\xi_{k}^{n+1}\right)\left(y^{n+1}-y_{k}^{n+1}\right)^{2}\right]+\sum_{i=1}^{p} \frac{\beta_{i}}{\beta_{0}} y^{n+1-i} .
$$

Now, by subtracting the identity (18) to (19), we obtain

$$
y^{n+1}-y_{k+1}^{n+1}=\frac{\Delta t}{\beta_{0}}\left[f_{y}\left(y_{k}^{n+1}\right)\left(y^{n+1}-y_{k+1}^{n+1}\right)+\frac{1}{2} f_{y y}\left(\xi_{k}^{n+1}\right)\left(y^{n+1}-y_{k}^{n+1}\right)^{2}\right]+\sum_{i=1}^{p} \frac{\beta_{i}}{\beta_{0}}\left(y^{n+1-i}-y_{J}^{n+1-i}\right),
$$

thus leading to

$$
\begin{aligned}
y^{n+1}-y_{k+1}^{n+1} & =\frac{1}{\beta_{0}-\Delta t f_{y}\left(y_{k}^{n+1}\right)}\left[\frac{1}{2} \Delta t f_{y y}\left(\xi_{k}^{n+1}\right)\left(y^{n+1}-y_{k}^{n+1}\right)^{2}+\sum_{i=1}^{p} \beta_{i}\left(y^{n+1-i}-y_{J}^{n+1-i}\right)\right] \\
& =C_{1, k} \Delta t\left(y^{n+1}-y_{k}^{n+1}\right)^{2}+\sum_{i=1}^{p} C_{2, i, k}\left(y^{n+1-i}-y_{J}^{n+1-i}\right),
\end{aligned}
$$

where, thanks to hypotheses (14), we have set

$$
\begin{aligned}
& C_{1, k}=C_{1}(n+1, k):=\frac{1}{2} \frac{f_{y y}\left(\xi_{k}^{n+1}\right)}{\beta_{0}-\Delta t f_{y}\left(y_{k}^{n+1}\right)} \leq \frac{1}{2} \frac{L_{2}}{\beta_{0}-\Delta t L_{1}}=: \widehat{C}_{1}, \\
& C_{2, i, k}=C_{2, i}(n+1, k):=\frac{\beta_{i}}{\beta_{0}-\Delta t f_{y}\left(y_{k}^{n+1}\right)} \leq \frac{\max _{i}\left|\beta_{i}\right|}{\beta_{0}-\Delta t L_{1}}=: \widehat{C}_{2} .
\end{aligned}
$$

We observe that hypothesis (15) guarantees that the previous quantities and thus identity (20) make sense. 
If we perform exactly $J$ iterations in (20) also at time step $n+1$, and setting $\eta^{n}=y^{n}-y_{J}^{n}$, we obtain

$$
\eta^{n+1}=\sum_{i=1}^{p} C_{2, i, J-1} \eta^{n+1-i}+C_{1, J-1} \Delta t R\left(t^{n+1}\right),
$$

where we have set

$$
R\left(t^{n}\right):=\left(y^{n}-y_{J-1}^{n}\right)^{2} .
$$

The recursive application of (20), leads to the following bound

$$
\begin{aligned}
\left|y^{n+1}-y_{k+1}^{n+1}\right| \leq & 2^{3\left(2^{k+1}-1\right)} \widehat{C}_{1}^{2\left(2^{k+1}-1\right)} \Delta t^{2^{k+1}-1}\left|y^{n+1}-y_{0}^{n+1}\right|^{2^{k+1}} \\
& +\sum_{l=0}^{k} 2^{2\left(2^{l+1}-1\right)} \widehat{C}_{1}^{2^{l+1}-1} \Delta t^{2^{l}-1}\left(\sum_{i=1}^{p} \widehat{C}_{2}\left(y^{n+1-i}-y_{J}^{n+1-i}\right)\right)^{2^{l}} .
\end{aligned}
$$

We also notice that

$$
\begin{aligned}
y^{n+1}-y_{0}^{n+1}= & y^{n+1}-E_{q}\left(\boldsymbol{y}_{J}^{n, q}\right)=y^{n+1}-E_{q}\left(\boldsymbol{y}_{J}^{n, q}\right) \pm E_{q}\left(\boldsymbol{y}^{n, q}\right) \pm y\left(t^{n+1}\right) \pm E_{q}\left(\boldsymbol{y}\left(t^{n, q}\right)\right) \\
= & \underbrace{\left(y^{n+1}-y\left(t^{n+1}\right)\right)}_{O\left(\Delta t^{p}\right)}+\underbrace{\left(y\left(t^{n+1}\right)-E_{q}\left(\boldsymbol{y}\left(t^{n, q}\right)\right)\right)}_{O\left(\Delta t^{q}\right)} \\
& +\underbrace{E_{q}\left(\boldsymbol{y}\left(t^{n, q}\right)-\boldsymbol{y}^{n, q}\right)}_{O\left(\Delta t^{p}\right)}+E_{q}\left(\boldsymbol{\eta}^{n, q}\right)=O\left(\Delta t^{q}\right)+E_{q}\left(\boldsymbol{\eta}^{n, q}\right),
\end{aligned}
$$

where in the last equality we have exploited that $q \leq p$. Then, thanks to (25) and (13), we obtain from (24)

$$
\begin{aligned}
\left|y^{n+1}-y_{k+1}^{n+1}\right| \leq & K_{1} \Delta t^{2^{k+1}-1}\left(K_{2} \Delta t^{2^{k+1} q}+K_{3}\left(\sum_{i=1}^{q}(-1)^{i+1}\left(\begin{array}{l}
q \\
i
\end{array}\right) \eta^{n+1-i}\right)^{2^{k+1}}\right) \\
& +K_{4} \sum_{l=0}^{k} \Delta t^{2^{l}-1}\left(\sum_{i=1}^{p} \widehat{C}_{2} \eta^{n+1-i}\right)^{2^{l}}=K_{5} \Delta t^{2^{k+1}(q+1)-1}+P_{2^{k+1}}\left(\eta^{n, p}\right),
\end{aligned}
$$

for suitable constants $K_{j}, j=1, \ldots, 5$, and where $P_{S}$ is a suitable polynomial of order $s$ with $P_{s}(0, \ldots, 0)=0$.

Now, thanks to the bound (26) evaluated for $k+1=J-1$, from (23) we have

$$
R\left(t^{n+1}\right)=\left(y^{n+1}-y_{J-1}^{n+1}\right)^{2} \leq 2 K_{5}^{2} \Delta t^{2^{J}(q+1)-2}+2\left(P_{2^{J-1}}\left(\eta^{n, p}\right)\right)^{2} .
$$

This shows that $R\left(t^{n+1}\right)$ does not depend on $\eta^{n+1}$. In particular, from (24) we can write

$$
R\left(t^{n+1}\right)=\left(y^{n+1}-y_{J-1}^{n+1}\right)^{2}=R\left(\eta^{n, p}, y^{n+1}-y_{0}^{n+1}\right),
$$

and thus from (25) we obtain

$$
R\left(t^{n+1}\right)=R\left(\eta^{n, p}, \varepsilon^{n+1, q+1}, \chi_{q}^{n+1}\right),
$$

where

$$
\varepsilon^{n}:=y\left(t^{n}\right)-y^{n}, \quad \chi_{q}^{n}:=y\left(t^{n}\right)-E_{q}\left(\boldsymbol{y}\left(t^{n-1, q}\right)\right) .
$$

In what follows we will refer to $R$ as either $R\left(t^{n+1}\right)$ or $R\left(\eta^{n, p}\right)$.

Now, exploiting the definitions (21), we can rewrite problem (22) as follows

$$
\beta_{0} \eta^{n+1}-\sum_{i=1}^{p} \beta_{i} \eta^{n+1-i}=\Delta t f_{y}\left(y_{J-1}^{n+1}\right) \eta^{n+1}+\frac{1}{2} \Delta t f_{y y}\left(\xi_{J-1}^{n+1}\right) R\left(\eta^{n, p}\right) .
$$

We now apply a fixed point strategy to the previous problem to show that its solution $\eta^{n, p}$ exists and is bounded. In particular, starting from (29) and assuming that the solutions $y^{n+1}$ and $y_{k}^{n+1}, k=0, \ldots, J$, exist, we consider the following problem for the error

$$
\beta_{0} \eta^{n+1}-\sum_{i=1}^{p} \beta_{i} \eta^{n+1-i}=\Delta t f_{y}\left(y_{J-1}^{n+1}\right) \eta^{n+1}+\frac{1}{2} \Delta t f_{y y}\left(\xi_{J-1}^{n+1}\right) R\left(\widetilde{\eta}^{n, p}\right),
$$


for a given $\widetilde{\eta}^{n, p}$. We can now apply Proposition 1 to problem (30), with $z^{n}=\eta^{n}, g_{1}\left(\eta^{n+1}\right)=f_{y}\left(y_{J-1}^{n+1}\right) \eta^{n+1}$ and $g_{2}\left(t^{n+1}\right)=$ $\frac{1}{2} f_{y y}\left(\xi_{J-1}^{n+1}\right) R\left(\widetilde{\boldsymbol{\eta}}^{n, p}\right)$, obtaining, thanks to (27) and hypothesis $(14)_{2}$,

$$
\begin{aligned}
\left|\eta^{n}\right| & \leq \frac{1}{2} K T \max _{j=p, \ldots, n}\left|f_{y y}\left(\xi_{J-1}^{j}\right) R\left(t^{j}\right)\right| \\
& \leq K T L_{2}\left(K_{5}^{2} \Delta t^{2^{J}(q+1)-2}+\max _{j=1, \ldots, n-p+1}\left(P_{2^{J-1}}\left(\widetilde{\eta}^{n-j, p}\right)\right)^{2}\right),
\end{aligned}
$$

where we have exploited the fact that $y^{i}=y_{J}^{i}$ are given for $i=0, \ldots, p-1$, so that $\eta^{i}=0$ for $i=0, \ldots, p-1$.

Now, we check that, if the sequence $\left(\widetilde{\eta}^{0}, \ldots, \widetilde{\eta}^{n}\right)$ verifies $\left|\widetilde{\eta}^{j}\right| \leq C \Delta t^{r}$, with $r=2^{J}(q+1)-2$ and suitable $C>0$, then the same holds for the solution $\left(\eta^{0}, \ldots, \eta^{n}\right)$. Indeed, from $(31)$ and recalling that $P_{2^{J-1}}(0, \ldots, 0)=0$, we have

$$
\left|\eta^{n}\right| \leq K T L_{2}\left(K_{5}^{2} \Delta t^{r}+K_{6} \Delta t^{2 r}\right)
$$

for a suitable $K_{6}=K_{6}(C)$ which is a polynomial of degree $2^{J-1}$ in $C$. Then, $\eta^{n}$ belongs to the ball of radius $C \Delta t^{r}$ provided that

$$
K T L_{2}\left(K_{5}^{2}+K_{6}(C) \Delta t^{r}\right) \leq C,
$$

that is for $\Delta t$ small enough and $C>K T L_{2} K_{5}^{2}$. Now, since $R$ is a polynomial in $\eta^{n, q}$, the fixed-point map in (30) is continuous, and thus we can apply the Brower's Theorem to problem (30), obtaining the existence of a solution $\left(\eta^{0}, \ldots, \eta^{n}\right)$ with $\left|\eta^{j}\right| \leq C \Delta t^{r}$ for any $j$.

Remark 1. Intuitively, thanks to the quadratic convergence of the Newton method, one could expect that the initial error $O\left(\Delta t^{q}\right)$ due to the extrapolation is squared at each iteration, thus obtaining, after $J$ iterations, an error which is $O\left(\Delta t^{2^{J} q}\right)$. However, from the theory of the Newton convergence, we know that the constant appearing at each squaring is the Lipschitz constant related to the function which we are seeking the roots of, see [31]. In our case such a constant is $O(\Delta t)$, thus explaining our result.

Remark 2. We observe from definitions (21), that the stability condition (15) is not needed whenever the one-sided bound $-L_{1} \leq f_{y}(z) \leq 0$ holds, instead of $(14)_{1}$. In the case of a PDE model $\partial_{t} y+K(y)=g$, with $K$ a non-linear differential operator, the previous condition would correspond to having a coercive tangent operator $K_{y}$.

We consider now the analysis of the approximate-Newton-J method. In particular, the term $f_{y}\left(y_{k}^{n+1}\right)$ in the Newton method (16) is replaced by a suitable approximation $\widehat{f}_{y}\left(y_{k}^{n+1}\right)$. We have the following

Theorem 2. Suppose that $f_{y}$ is Lipschitz-continuous and globally bounded, and that

$$
\left|\widehat{f}_{y}(z)\right| \leq L_{3}, \quad\left|f_{y}(z)-\widehat{f}_{y}(z)\right|<M,
$$

for any $z$ and for suitable constants $L_{3}, M<+\infty$. Then, assuming that the solution $y^{n}$ exists for all $n$, if $M$ is small enough, there exists $\Delta t_{0}$ with

$$
\Delta t_{0}<\frac{\beta_{0}}{L_{3}}
$$

such that the BDFp method applied to (10) used in combination with the approximate-Newton-J method to solve Eq. (12) and with an extrapolation of order $q \leq p$ as initial guess at each time step, is globally of order

$$
s=\min \{p ; J+q-1\}
$$

for $\Delta t \leq \Delta t_{0}$.

Proof. Proceeding as for the exact Newton case, the approximate-Newton method applied to Eq. (12) leads to

$$
y_{k+1}^{n+1}=\frac{\Delta t}{\beta_{0}}\left[f\left(y_{k}^{n+1}\right)+\widehat{f}_{y}\left(y_{k}^{n+1}\right)\left(y_{k+1}^{n+1}-y_{k}^{n+1}\right)\right]+\sum_{i=1}^{p} \frac{\beta_{i}}{\beta_{0}} y^{n+1-i} .
$$

If we suppose to have performed at the previous time steps only $J$ iterations, the previous equality becomes

$$
y_{k+1}^{n+1}=\frac{\Delta t}{\beta_{0}}\left[f\left(y_{k}^{n+1}\right)+\widehat{f}_{y}\left(y_{k}^{n+1}\right)\left(y_{k+1}^{n+1}-y_{k}^{n+1}\right)\right]+\sum_{i=1}^{p} \frac{\beta_{i}}{\beta_{0}} y_{J}^{n+1-i} .
$$


Moreover, by writing the Taylor expansion around $y_{k}^{n+1}$, we obtain

$$
f\left(y^{n+1}\right)=f\left(y_{k}^{n+1}\right)+f_{y}\left(\xi_{k}^{n+1}\right)\left(y^{n+1}-y_{k}^{n+1}\right),
$$

for a suitable $\xi_{k}^{n+1} \in\left(\min \left\{y^{n+1} ; y_{k}^{n+1}\right\}, \max \left\{y^{n+1} ; y_{k}^{n+1}\right\}\right)$. Substituting the expression of $f\left(y^{n+1}\right)$ in (11) in the previous equality, we have

$$
y^{n+1}=\frac{\Delta t}{\beta_{0}}\left[f\left(y_{k}^{n+1}\right)+f_{y}\left(\xi_{k}^{n+1}\right)\left(y^{n+1}-y_{k}^{n+1}\right)\right]+\sum_{i=1}^{p} \frac{\beta_{i}}{\beta_{0}} y^{n+1-i} .
$$

Now, by adding and subtracting the term $\widehat{f}_{y}\left(y_{k}^{n+1}\right)\left(y^{n+1}-y_{k}^{n+1}\right)$ in (34), and by subtracting this new identity to (35), we obtain

$$
\begin{aligned}
y^{n+1}-y_{k+1}^{n+1}= & \frac{\Delta t}{\beta_{0}}\left[\left(f_{y}\left(\xi_{k}^{n+1}\right)-\widehat{f}_{y}\left(y_{k}^{n+1}\right)\right)\left(y^{n+1}-y_{k}^{n+1}\right)+\widehat{f}_{y}\left(y_{k}^{n+1}\right)\left(y^{n+1}-y_{k+1}^{n+1}\right)\right] \\
& +\sum_{i=1}^{p} \frac{\beta_{i}}{\beta_{0}}\left(y^{n+1-i}-y_{J}^{n+1-i}\right) .
\end{aligned}
$$

By adding and subtracting in the previous equality the quantity $f_{y}\left(y_{k}^{n+1}\right)\left(y^{n+1}-y_{k}^{n+1}\right)$, we obtain

$$
\begin{aligned}
y^{n+1}-y_{k+1}^{n+1}= & \frac{\Delta t}{\beta_{0}}\left[\left(f_{y}\left(y_{k}^{n+1}\right)-\widehat{f}_{y}\left(y_{k}^{n+1}\right)\right)\left(y^{n+1}-y_{k}^{n+1}\right)+\left(f_{y}\left(\xi_{k}^{n+1}\right)-f_{y}\left(y_{k}^{n+1}\right)\right)\left(y^{n+1}-y_{k}^{n+1}\right)\right. \\
& \left.+\widehat{f}_{y}\left(y_{k}^{n+1}\right)\left(y^{n+1}-y_{k+1}^{n+1}\right)\right]+\sum_{i=1}^{p} \frac{\beta_{i}}{\beta_{0}}\left(y^{n+1-i}-y_{J}^{n+1-i}\right) .
\end{aligned}
$$

This leads to

$$
\begin{aligned}
y^{n+1}-y_{k+1}^{n+1}= & \frac{1}{\beta_{0}-\Delta t \widehat{f}_{y}\left(y_{k}^{n+1}\right)}\left[\Delta t\left(f_{y}\left(y_{k}^{n+1}\right)-\widehat{f}_{y}\left(y_{k}^{n+1}\right)\right)\left(y^{n+1}-y_{k}^{n+1}\right)\right. \\
& +\Delta t\left(f_{y}\left(\xi_{k}^{n+1}\right)-f_{y}\left(y_{k}^{n+1}\right)\right)\left(y^{n+1}-y_{k}^{n+1}\right) \\
& \left.+\sum_{i=1}^{p} \beta_{i}\left(y^{n+1-i}-y_{J}^{n+1-i}\right)\right] \\
= & C_{1, k} \Delta t\left(y^{n+1}-y_{k}^{n+1}\right)+C_{2, k} \Delta t\left(y^{n+1}-y_{k}^{n+1}\right)^{2}+\sum_{i=1}^{p} C_{3, i, k}\left(y^{n+1-i}-y_{J}^{n+1-i}\right),
\end{aligned}
$$

where we have set, thanks to hypotheses (32),

$$
\begin{aligned}
& C_{1, k}=C_{1}(n+1, k):=\frac{f_{y}\left(y_{k}^{n+1}\right)-\widehat{f}_{y}\left(y_{k}^{n+1}\right)}{\beta_{0}-\Delta t \widehat{f}_{y}\left(y_{k}^{n+1}\right)} \leq \frac{M}{\beta_{0}-\Delta t L_{3}}=: \widehat{C}_{1}, \\
& C_{2, k}=C_{2}(n+1, k):=\frac{f_{y}\left(\xi_{k}^{n+1}\right)-f_{y}\left(y_{k}^{n+1}\right)}{\left(y^{n+1}-y_{k}^{n+1}\right)\left(\beta_{0}-\Delta t \widehat{f}_{y}\left(y_{k}^{n+1}\right)\right)} \leq \frac{L_{4}}{\beta_{0}-\Delta t L_{3}}=: \widehat{C}_{2}, \\
& C_{3, i, k}=C_{3, i}(n+1, k):=\frac{\beta_{i}}{\beta_{0}-\Delta t \widehat{f}_{y}\left(y_{k}^{n+1}\right)} \leq \frac{\max _{i}\left|\beta_{i}\right|}{\beta_{0}-\Delta t L_{3}}=: \widehat{C}_{3},
\end{aligned}
$$

and where $L_{4}$ is the Lipschitz constant related to $f_{y}$. We observe that the assumptions (32) and (33) guarantee that the previous quantities and thus identity (36) make sense.

If we perform exactly $J$ iterations in (36) also at time step $n+1$, and setting again $\eta^{n}=y^{n}-y_{J}^{n}$, we obtain

$$
\eta^{n+1}=\sum_{i=1}^{p} C_{3, i, J-1} \eta^{n+1-i}+\Delta t C_{1, J-1} S\left(t^{n+1}\right)+\Delta t C_{2, J-1}\left(S\left(t^{n+1}\right)\right)^{2},
$$

where we have set

$$
S\left(t^{n}\right)=y^{n}-y_{J-1}^{n} \text {. }
$$

Proceeding as in Theorem 1 (we skip the details for the sake of exposition), the recursive application of (36) leads, thanks to (25) and (13), to the following bound 


$$
\left|y^{n+1}-y_{k+1}^{n+1}\right| \leq K_{1} \Delta t^{k+q+1}+P_{2^{k+1}}\left(\eta^{n, p}\right),
$$

for a suitable constant $K_{1}$, and where again $P_{2^{k+1}}(0, \ldots, 0)=0$.

Now, thanks to the bound (40) evaluated for $k+1=J-1$, from (39) we have

$$
S\left(t^{n+1}\right)=y^{n+1}-y_{J-1}^{n+1} \leq K_{1} \Delta t^{J+q-1}+P_{2^{J-1}}\left(\eta^{n, p}\right) .
$$

This shows that $S\left(t^{n+1}\right)$ does not depend on $\eta^{n+1}$, and in particular we can write

$$
S\left(t^{n+1}\right)=S\left(\eta^{n, p}, y^{n+1}-y_{0}^{n+1}\right)=S\left(\eta^{n, p}, \boldsymbol{\varepsilon}^{n+1, q+1}, \chi_{q}^{n+1}\right),
$$

where $\varepsilon^{n}$ and $\chi_{q}^{n}$ are defined in (28). In what follows we will refer to $S$ as either $S\left(t^{n+1}\right)$ or $S\left(\eta^{n, p}\right)$.

Now, exploiting the definitions (37), we can rewrite problem (38) as follows

$$
\begin{aligned}
\beta_{0} \eta^{n+1}-\sum_{i=1}^{p} \beta_{i} \eta^{n+1-i}= & \Delta t \widehat{f}_{y}\left(y_{J-1}^{n+1}\right) \eta^{n+1}+\Delta t\left(f_{y}\left(y_{J-1}^{n+1}\right)-\widehat{f}_{y}\left(y_{J-1}^{n+1}\right)\right) S\left(\eta^{n, p}\right) \\
& +\Delta t \frac{f_{y}\left(\xi_{J-1}^{n+1}\right)-f_{y}\left(y_{J-1}^{n+1}\right)}{y^{n+1}-y_{J-1}^{n+1}}\left(S\left(\eta^{n, p}\right)\right)^{2} .
\end{aligned}
$$

We now apply a fixed point strategy to the previous problem to show that its solution $\eta^{n, p}$ exists and is bounded. In particular, starting from (38) and assuming that the solutions $y^{n+1}$ and $y_{k}^{n+1}, k=0, \ldots, J$, exist, we consider the following problem for the error

$$
\begin{aligned}
\beta_{0} \eta^{n+1}-\sum_{i=1}^{p} \beta_{i} \eta^{n+1-i}= & \Delta t \widehat{f}_{y}\left(y_{J-1}^{n+1}\right) \eta^{n+1}+\Delta t\left(f_{y}\left(y_{J-1}^{n+1}\right)-\widehat{f}_{y}\left(y_{J-1}^{n+1}\right)\right) S\left(\widetilde{\boldsymbol{\eta}}^{n, p}\right) \\
& +\Delta t \frac{f_{y}\left(\xi_{J-1}^{n+1}\right)-f_{y}\left(y_{J-1}^{n+1}\right)}{y^{n+1}-y_{J-1}^{n+1}}\left(S\left(\widetilde{\boldsymbol{\eta}}^{n, p}\right)\right)^{2},
\end{aligned}
$$

for a given $\widetilde{\eta}^{n, p}$. We can now apply Proposition 1 to problem (42), with $z^{n}=\eta^{n}, g_{1}\left(\eta^{n+1}\right)=\widehat{f}_{y}\left(y_{J-1}^{n+1}\right) \eta^{n+1}$ and $g_{2}\left(t^{n+1}\right)$ given by the second and third term at the right hand side in (42), obtaining, thanks to (41) and definitions (37),

$$
\begin{aligned}
\left|\eta^{n}\right| & \leq K T \max _{j=p, \ldots, n}\left|\left(f_{y}\left(y_{J-1}^{j}\right)-\widehat{f}_{y}\left(y_{J-1}^{j}\right)\right) S\left(t^{j}\right)+\frac{f_{y}\left(\xi_{J-1}^{j}\right)-f_{y}\left(y_{J-1}^{j}\right)}{y^{j}-y_{J-1}^{j}}\left(S\left(t^{j}\right)\right)^{2}\right| \\
& \leq K T \max _{j=1, \ldots, n-p+1}\left|M\left(K_{1} \Delta t^{J+q-1}+P_{2^{J-1}}\left(\widetilde{\boldsymbol{\eta}}^{n-j, p}\right)\right)+L_{4}\left(K_{1} \Delta t^{J+q-1}+P_{2^{J-1}}\left(\widetilde{\boldsymbol{\eta}}^{n-j, p}\right)\right)^{2}\right|,
\end{aligned}
$$

where we have exploited again that $\eta^{i}=0$ for $i=0, \ldots, p-1$.

Now, we check that if the sequence $\left(\widetilde{\eta}^{0}, \ldots, \widetilde{\eta}^{n}\right)$ verifies $\left|\widetilde{\eta}^{j}\right| \leq C \Delta t^{s}$, with $s=J+q-1$ and suitable $C>0$, then the same holds for the solution $\left(\eta^{0}, \ldots, \eta^{n}\right)$. Indeed, recalling that $P_{2^{J-1}}(0, \ldots, 0)=0$ so that $\left|P_{2^{J-1}}\left(\widetilde{\eta}^{n, p}\right)\right| \leq K_{2}(C) \Delta t^{s}$ for a suitable $K_{2}$ which is a polynomial of degree $2^{J-1}$ in $C$, from (43) we have

$$
\left|\eta^{n}\right| \leq K T \Delta t^{s}\left(K_{1}\left(M+2 L_{4} K_{1} \Delta t^{s}\right)+K_{2}(C)\left(M+2 L_{4} K_{2}(C) \Delta t^{s}\right)\right) .
$$

Then, $\eta^{n}$ belongs to the ball of radius $C \Delta t^{s}$ provided that

$$
K T\left(K_{1}\left(M+2 L_{4} K_{1} \Delta t^{s}\right)+K_{2}(C)\left(M+2 L_{4} K_{2}(C) \Delta t^{s}\right)\right) \leq C,
$$

that is for $\Delta t$ and $M$ sufficiently small (i.e. for a good approximation of the Jacobian). Now, by exploiting the continuity of the fixed-point map in (42), we can apply the Brower's Theorem to problem (42), obtaining the existence of a solution $\left(\eta^{0}, \ldots, \eta^{n}\right)$ with $\left|\eta^{j}\right| \leq C \Delta t^{s}$ for any $j$, provided that the approximation of the Jacobian is accurate enough.

Remark 3. The FSI problem is a system of partial differential equations involving first and second order time derivative and can be written as a first order system. Its time discretization leads to a system of non-linear equations, whereas our analysis have been written for the scalar case. Therefore, to extend our analysis to the FSI problem, one needs to use vector and matrix norms, making the proofs quite complicate and technical. However, we believe that the first order system nature of the FSI problem allows us to apply our theoretical results to such a problem, and this is confirmed by the numerical results presented in what follows.

In Table 1 we report the predicted order of the proposed methods with respect to the solution obtained by solving until convergence the Newton or the approximate-Newton methods. This table gives us precise information about the BDF 
Table 1

Order of the error $E_{J}$ for different values of the number of iterations $J$ and of the order of extrapolation $q$.

\begin{tabular}{|c|c|c|c|c|c|c|}
\hline \multirow[t]{2}{*}{$J / q$} & \multicolumn{3}{|c|}{ Newton-J } & \multicolumn{3}{|c|}{ Approximate Newton-J } \\
\hline & 1 & 2 & 3 & 1 & 2 & 3 \\
\hline 1 & 2 & 4 & 6 & 1 & 2 & 3 \\
\hline 2 & 6 & 10 & 14 & 2 & 3 & 4 \\
\hline 3 & 14 & 22 & 30 & 3 & 4 & 5 \\
\hline
\end{tabular}

schemes which could be used in combination with a particular choice of $J$ and $q$. We observe, for example, that by performing only 1 iteration and by using an extrapolation of the first order as initial guess, the Newton-J allows to recover a second order scheme when used in combination with a BDFp method, $p \geq 2$, whereas the approximate-Newton-J allows to recover a first order method when used in combination with a BDFp method, $p \geq 1$. Instead, if we perform 2 iterations and use a first order extrapolation, we are able to recover order 6 for the Newton-J method and order 2 for the approximate-Newton-J method. Finally, we observe that for the approximate-Newton-J method, which is the case we are interested in, each iteration allows us to gain an order in $\Delta t$ up to the maximum order $p$ of the BDFp scheme.

\section{The exact case: an efficient choice of the internal tolerance}

From Algorithm 1 we can recover the exact Double-loop scheme GC $\infty$-P $\infty$ by allowing the number of external iterations to be enough to reach convergence over the geometrical interface conditions and the constitutive non-linearities $(m=+\infty)$. In such a case, for the external loop, we have to monitor the residual of condition $(6)_{2}$ and the residuals related to the convergence of the non-linearities in the fluid and in the structure subproblems.

Now, we observe that when a Newton or an approximate-Newton method is considered to solve a non-linear system of equations, the linear system involved at each iteration does not need to be solved until convergence when an iterative method is considered for its solution, unless the external Newton residual has not become small. Indeed, as observed for example in [31], it is enough to stop the internal iterations when the residual is below a tolerance proportional to the Newton residual. This leads to a great saving in the computational times, without affecting the accuracy, since at convergence of the Newton iterations the tolerance of the internal linear system has become sufficiently small.

It is then possible to apply such an idea to our case, that is to the $\mathrm{GC} \infty-\mathrm{P} \infty$ scheme. In such a case, we conclude that the FSI linear system arising at each approximate-Newton iteration (step 2. in Algorithm 1) does not need to be solved until convergence. This means that the physical interface conditions does not need in fact to be satisfied at each approximate-Newton iteration. In particular, the tolerance of the internal loop is taken proportional to the external residual

$$
\mathcal{R}^{k}:=\left\|\widetilde{\gamma}_{s} \widetilde{\boldsymbol{\eta}}_{s}^{k}-\widetilde{\gamma}_{s} \widetilde{\boldsymbol{\eta}}_{s}^{k-1}\right\|+\left\|\left(\left(\boldsymbol{u}_{f}^{k}-\boldsymbol{u}_{f}^{k-1}\right) \cdot \nabla\right) \boldsymbol{u}_{f}^{k}\right\|+\left\|\mathcal{G}_{s}-\mathcal{S}\left(\widetilde{\boldsymbol{\eta}}_{s}^{k}\right)-\widetilde{\gamma}_{s}^{*} \widetilde{\lambda}_{s}^{k}\right\|,
$$

where suitable norms are used in each term of (44). The first term is the residual of the geometrical interface condition, the second one is the residual related to the fluid non-linearity and the third one is the residual related to the structure non-linearity. Therefore, at convergence of the approximate-Newton loop, the physical interface conditions are satisfied too, so that this scheme is in fact exact. Observe that such an idea could not be applied to the inexact algorithms $(m<\infty)$, because we would not guarantee the satisfaction of the physical interface conditions, since the internal tolerance would not become small enough.

In [31] such a strategy has been referred to as inexact-Newton. However, in order to avoid confusion with the inexact schemes presented in this work, we name these algorithms exact schemes with dynamic tolerance and we add the suffix DT at the end of the name of the algorithm. In Algorithm 2, we detail the GC $\infty$-P $\infty$-DT scheme.

For the choice of $\sigma^{k}$ at step 2 we follow [31]. In particular, we set

$$
\sigma^{k}= \begin{cases}\sigma_{\max } & k=0, \\ \min \left(\sigma_{\max }, \gamma\left(\mathcal{R}^{k} / \mathcal{R}^{k-1}\right)^{2}\right) & k>0, \gamma\left(\sigma^{k-1}\right)^{2} \leq 0.1, \\ \min \left(\sigma_{\max }, \max \left(\gamma\left(\mathcal{R}^{k} / \mathcal{R}^{k-1}\right)^{2}, \gamma\left(\sigma^{k-1}\right)^{2}\right)\right) & k>0, \gamma\left(\sigma^{k-1}\right)^{2}>0.1 .\end{cases}
$$

In the numerical simulations presented in this work we have used $\sigma_{\max }=0.9999$ and $\gamma=0.9$. In practical implementations of the residuals (44) and (45) we considered the $L^{2}$ norms.

Remark 4. In [31] it has been shown that the choice (46) guarantees a second order convergence when the exact Jacobian is used in the iterations. For approximate-Newton strategies, as in our case, this choice allows to recover at least first order of convergence.

Another exact scheme of this family, obtained by considering a different approximation of the Jacobian, is the so-called Single-loop (GCP $\infty)$ scheme [27,36,33,41], where all the non-linearities and interface conditions are treated in the same loop. Single-loop scheme could be obtained by Algorithm 1 with $m=+\infty$ and by performing just 1 internal iteration, however monitoring the residual of condition $(7)_{2}$. 


\section{Algorithm 2 GC $\infty$-P $\infty$-DT scheme.}

Given the solutions $\tilde{\boldsymbol{\eta}}_{s}^{k}$ and $\boldsymbol{u}_{f}^{k}$ and $0<\omega \leq 1$

UNTIL G-C convergence Do

1. Compute $\widetilde{\eta}_{m}^{k+1}$ and $\tilde{\lambda}_{m}^{k+1}$ by solving the harmonic extension for the fluid domain (6), obtaining the new fluid domain $\Omega_{f}^{k+1}$ and the fluid domain velocity $\boldsymbol{u}_{m}^{k+1}$ by using (4);

2. Solve the linearized FSI problem. For its solution, given the external residual $\mathcal{R}^{k}$ defined by (44), we consider the following partitioned algorithm:

Given the solution at subiteration $l-1$ and a suitable scalar $\sigma^{k+1}$,

UNTIL

$$
\left\|\frac{\alpha_{f} \beta_{0}}{\Delta t}\left(\widetilde{\gamma}_{s} \widetilde{\boldsymbol{\eta}}_{s, l}^{k+1}-\widetilde{\gamma}_{s} \widetilde{\boldsymbol{\eta}}_{s, l-1}^{k+1}\right)+\tilde{\lambda}_{s, l}^{k+1}-\widetilde{\lambda}_{s, l-1}^{k+1}\right\| \geq \sigma^{k} \mathcal{R}^{k},
$$

Do

(a) Compute $\boldsymbol{v}_{f, l}^{k+1}$ and $\tilde{\lambda}_{f, l}^{k+1}$ by solving the linearized fluid subproblem (7)

(b) Compute $\widetilde{\boldsymbol{\eta}}_{s, l}^{k+1}$ and $\widetilde{\lambda}_{s, l}^{k+1}$ by solving the linearized structure subproblem (8)

(c) Relaxation step (9);

(d) Update: $l \rightarrow l+1$.

END DO

3. Update: $k \rightarrow k+1$.

\section{END DO}

\section{Numerical results}

In all the numerical experiments of this work, we considered the nearly incompressible exponential material whose first Piola-Kirchhoff tensor reads

$$
\widetilde{\boldsymbol{T}}_{S}\left(\boldsymbol{F}_{S}\right)=G J_{S}^{-2 / 3}\left(\boldsymbol{F}_{S}-\frac{1}{3} \operatorname{tr}\left(\boldsymbol{F}_{S}^{T} \boldsymbol{F}_{S}\right) \boldsymbol{F}_{S}^{-T}\right) e^{\gamma\left(J_{S}^{-\frac{2}{3}} \operatorname{tr}\left(\boldsymbol{F}_{S}^{T} \boldsymbol{F}_{S}\right)-3\right)}+\frac{\kappa}{2}\left(J_{s}-1+\frac{1}{J} \ln \left(J_{S}\right)\right) J_{S} \boldsymbol{F}_{S}^{-T} .
$$

Here $\boldsymbol{F}_{s}:=\nabla_{\boldsymbol{x}_{s}^{0}} \boldsymbol{x}_{s}^{t}$, where $\boldsymbol{x}_{s}^{0}$ are the coordinates in the reference configuration and $\boldsymbol{x}_{s}^{t}$ those in the current configuration, $J_{S}:=\operatorname{det}\left(\boldsymbol{F}_{S}\right), \kappa$ is the bulk modulus and $G$ the shear modulus. For small deformations such material behaves as a linear structure described by the infinitesimal elasticity, characterized by a Poisson's ratio $\nu$ and a Young modulus $E$ related to $\kappa$ and $G$ as follows

$$
\kappa=\frac{E}{3(1-2 v)}, \quad G=\frac{E}{2(1+v)} .
$$

The parameter $\gamma$ in (47) characterizes the stiffness of the material for large displacements.

We used P1bubble - P1 finite elements for the fluid subproblem and $P 1$ finite elements for the structure subproblem, and the following data: viscosity $\mu=0.03 \mathrm{~g} /(\mathrm{cms})$, fluid density $\rho_{f}=1 \mathrm{~g} / \mathrm{cm}^{3}$, structure density $\rho_{s}=1.2 \mathrm{~g} / \mathrm{cm}^{3}$, bulk modulus $\kappa=10^{7}$ dyne $/ \mathrm{cm}^{2}$, shear modulus $G=1.034 \cdot 10^{6}$ dyne $/ \mathrm{cm}^{2}$ (corresponding for small displacements to $E=3$. $10^{6}$ dyne $/ \mathrm{cm}^{2}$ and $\left.v=0.45\right), \gamma=1$. Moreover, if not otherwise specified, we used as time discretization parameter $\Delta t=$ $0.002 \mathrm{~s}$. This value has been chosen in order to guarantee that the exact schemes provide a stable and physically significant solution. Then, the same value has been used also for the inexact schemes, providing again stable results. We did not experienced further stability problems induced by the inexactness of the new schemes. A systematic study of their stability will be addressed in future works.

For the prescription of the interface continuity conditions, in all the simulations we have considered the Robin-Robin (RR) scheme [3,4], with the optimized coefficients proposed in [26] and adapted to the various temporal schemes in [41]. In all the simulations of this work, RR scheme has converged without any relaxation $(\omega=1)$, confirming its suitability for haemodynamic applications. The numerical experiments have been performed with the parallel Finite Element library LIFEV (www. lifev.org), see [41] for details.

\subsection{Efficiency of exact schemes in two real cases}

In the simulations of this section and of Section 5.3 we considered the computational domains depicted in Fig. 1, representing the carotid of a patient after the removal of the atherosclerotic plaque, and in Fig. 2, representing the abdominal aorta (AA) in a patient characterized by the presence of an aneurysm. The vessel lumen has been reconstructed by using the code VMTK (see www. vmtk. org), while the structure geometry has been obtained by extrusion, setting the ratio between the lumen radius and the thickness equal to 0.24 . The number of degrees of freedom is about 53000 (carotid) and 380000 (AA) for the fluid velocity, about 10000 (carotid) and 43000 (AA) for the fluid pressure and about 24000 (carotid) and 72000 (AA) for the structure displacement, and the fluid and structure meshes are conforming at the interface. For the harmonic extension and for the structure, we prescribed at the artificial sections normal homogeneous Dirichlet conditions and 

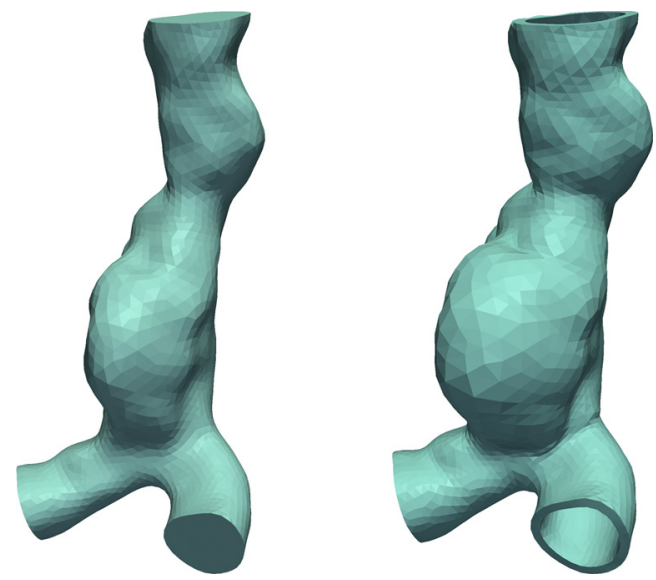

Fig. 2. Computational fluid (left) and structure (right) models of the abdominal aorta.
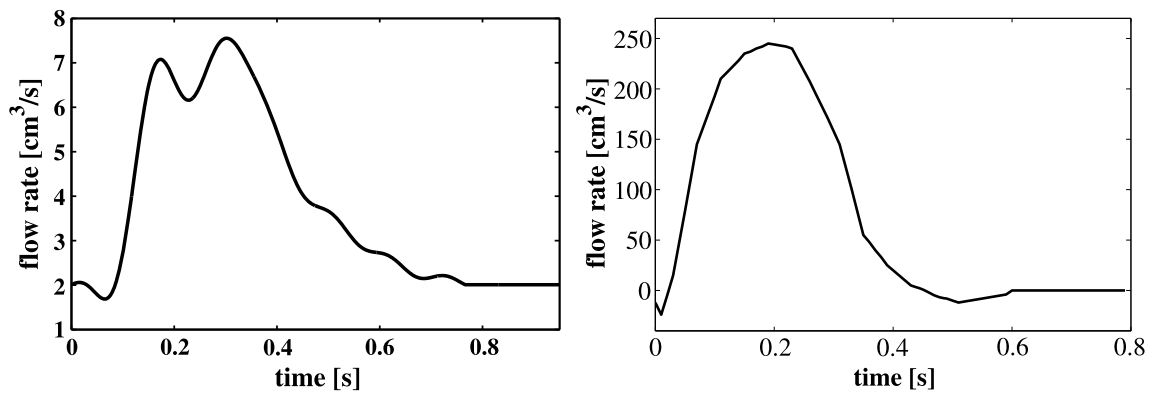

Fig. 3. Patient-specific flow rate waveforms prescribed at the inlet of the carotid (left) and of the abdominal aorta (right).

tangential homogeneous Neumann conditions, that is we let the domain move freely in the tangential direction. At the fluid inlet we imposed the patient-specific flow rates depicted in Fig. 3, left, for the carotid, and in Fig. 3, right, for the abdominal aorta. In both cases, this datum has been prescribed through the Lagrange multipliers method (see $[19,49,22,23])$. At the fluid outlets, we used an absorbing resistance boundary condition, see [42,50,41] for details. At the external surface $\Sigma_{\text {out }}^{0}$ we prescribed a Robin boundary condition with Robin coefficient $\alpha_{e}$ with the aim of modeling the presence of a surrounding tissue around the vessel $[35,38,10,41]$. In particular, we set $\alpha_{e}=3 \cdot 10^{6}$ dyne $/ \mathrm{cm}^{3}$. This value allows to recover a pressure in the physiological range.

Here, we want to compare the performance of different exact schemes. In particular, we considered GC $\infty-\mathrm{P} \infty$, $\mathrm{GC} \infty-\mathrm{P} \infty$-DT and GCP $\infty$ schemes together with classical algorithms. Among them, we considered GP $\infty-\mathrm{C} \infty$ scheme, corresponding to simple iterations at each time step between the fluid geometry and the fluid and the structure subproblems (see $[28,32,34,30]$ for the Dirichlet-Neumann case). Here, we considered the Robin-Robin version of such schemes introduced in [43]. At each iteration of the latter algorithm, the fluid and structure subproblems have to be solved with a proper strategy to handle the constitutive non-linearities. Here, we considered Picard iterations for the fluid and Newton iterations for the structure. We observe that in such a scheme the geometrical interface condition is treated together with the physical interface conditions. We also considered the treatment of the geometrical interface condition in an external loop, leading to an algorithm characterized by a triple loop nature $(\mathrm{G} \infty-\mathrm{P} \infty-\mathrm{C} \infty$ scheme).

As a representative case, we reported in Fig. 4 two snapshots of the streamlines at the sistole obtained with GC $\infty-\mathrm{P} \infty$ scheme and BDF1/BDF1 time discretization.

In Table 2 we reported the number of iterations for these schemes. The number of external iterations reported in the table has to be interpreted as the average one over one heart beat, whilst the intermediate and the internal ones as the average per outer loop (the external and the intermediate ones, respectively). We reported also the CPU time normalized over that of GC $\infty$-P $\infty$-DT scheme, used here as our gold-standard.

Discussion of the numerical results. The results reported in Table 2 show an optimal qualitative agreement between the performance obtained in the two geometries. They show that the approximate-Newton-based schemes are the most efficient among exact methods. In particular, we found that $\mathrm{GC} \infty-\mathrm{P} \infty$ scheme is slightly faster than $\mathrm{GCP} \infty$ and that the $\mathrm{CPU}$ time needed by GC $\infty$-P $\infty$-DT have been almost halved with respect to GC $\infty$-P $\infty$. Concerning the classical schemes, they showed a very poor efficiency in comparison to approximate-Newton schemes, their CPU time being more than three times greater with respect to that of $\mathrm{GC} \infty-\mathrm{P} \infty$-DT. Such schemes are however the most appealing from the implementation point of 

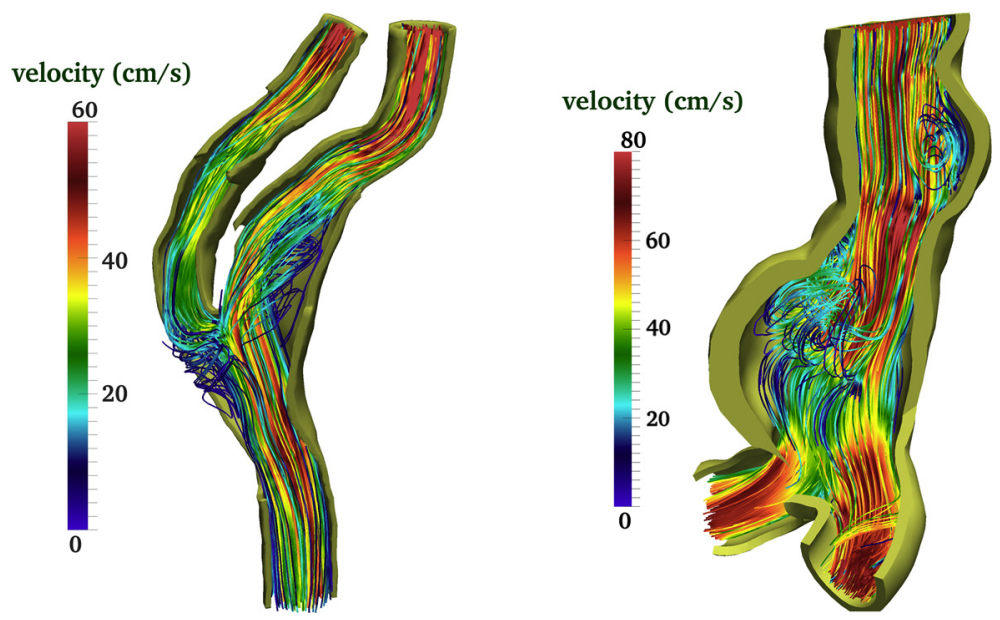

Fig. 4. Streamlines of the velocity field at the sistole in the carotid (left) and in the abdominal aorta (right). GC $\infty-\mathrm{P} \infty-\mathrm{BDF} 1 / \mathrm{BDF} 1$.

Table 2

Average number of iterations in the external loop and average number of iterations per outer loop in the intermediate and internal loops, and CPU time normalized with respect to that of GC $\infty$-P $\infty$-DT scheme. Exact schemes. BDF1/BDF1. For each box, left: carotid; right: abdominal aorta.

\begin{tabular}{|c|c|c|c|c|c|c|c|c|}
\hline \multirow[b]{2}{*}{$\mathrm{GC} \infty-\mathrm{P} \infty$} & \multicolumn{2}{|c|}{$\begin{array}{l}\text { \# of external } \\
\text { iterations }\end{array}$} & \multicolumn{2}{|c|}{$\begin{array}{l}\text { \# of intermediate } \\
\text { iterations }\end{array}$} & \multicolumn{2}{|c|}{$\begin{array}{l}\text { \# of internal } \\
\text { iterations }\end{array}$} & \multicolumn{2}{|c|}{$\begin{array}{l}\text { Normalized } \\
\text { CPU time }\end{array}$} \\
\hline & 13.5 & 8.9 & - & - & 7.7 & 7.6 & 1.72 & 1.69 \\
\hline GC $\infty-\mathrm{P} \infty-\mathrm{DT}$ & 13.9 & 9.4 & - & - & 3.0 & 2.9 & 1.00 & 1.00 \\
\hline $\mathrm{GCP} \infty$ & 20.4 & 17.4 & - & - & - & - & 2.00 & 2.04 \\
\hline $\mathrm{G} \infty-\mathrm{P} \infty-\mathrm{C} \infty$ & 12.9 & 8.5 & 6.7 & 6.5 & 3.5 & 3.5 & 3.76 & 3.35 \\
\hline $\mathrm{GP} \infty-\mathrm{C} \infty$ & 15.3 & 11.4 & - & - & 3.8 & 3.8 & 4.16 & 3.97 \\
\hline
\end{tabular}

view, when one has at disposal two black-box solvers for the fluid problem in ALE formulation and for the structure, since one would need just to implement suitable routines to transfer the interface information between the two codes. Instead, approximate-Newton-based schemes can be implemented in a modular way provided that one can access the fluid and structure tangent problems (always possible by running just one Newton iteration).

In conclusion, we found that, at least for the two real cases considered in this work, GC $\infty-\mathrm{P} \infty$-DT is the most suitable among the exact schemes presented here for real haemodynamic applications.

\subsection{Numerical results for the inexact schemes: convergence with respect to time}

In this section we want to validate the theoretical results developed in Section 3.1 by considering a 3D FSI problem. To this aim, we used the analytical test case proposed in [41] for the linear infinitesimal elasticity. This test consists in a translation of a cylinder of small thickness (the structure) filled by the fluid and in a rotation around its axis. Referring to the same data reported in [41], it is easy to check that the analytical solution of such a test is given by

$$
\left\{\begin{array}{llll}
\boldsymbol{u}_{f}=\overline{\boldsymbol{u}} & \text { in } \Omega_{f}^{t}, & p_{f}=0 & \text { in } \Omega_{f}^{t}, \\
\tilde{\boldsymbol{\eta}}_{s}=\overline{\boldsymbol{\eta}} & \text { in } \Omega_{s}^{0}, & \tilde{\boldsymbol{\eta}}_{m}=\overline{\boldsymbol{\eta}} & \text { in } \Omega_{f}^{0},
\end{array}\right.
$$

where

$$
\overline{\boldsymbol{\eta}}:=\left[\begin{array}{l}
x_{s, 1}^{0}(\cos \theta-1)-x_{s, 2}^{0} \sin \theta+c_{1}, \\
x_{s, 1}^{0} \sin \theta+x_{s, 2}^{0}(\cos \theta-1)+c_{2}, \\
c_{3},
\end{array}\right], \quad \overline{\boldsymbol{u}}:=\left[\begin{array}{l}
\dot{\theta}\left(c_{2}-x_{f, 2}\right)+\dot{c}_{1}, \\
\dot{\theta}\left(x_{f, 1}-c_{1}\right)+\dot{c}_{2}, \\
\dot{c}_{3},
\end{array}\right]
$$

for given functions of time $\theta(t)$ and $\boldsymbol{c}(t)$ and for suitable forcing terms.

We considered, in particular, the cylindrical geometry depicted in Fig. 5, with length $L=5 \mathrm{~cm}$, fluid domain radius $R=0.5 \mathrm{~cm}$, structure thickness $H_{s}=0.1 \mathrm{~cm}$. The space discretization parameter is $h=0.025 \mathrm{~cm}$ and the fluid and structure meshes are conforming at the interface. The number of degrees of freedom is about 16000 for the fluid velocity, about 2000 for the fluid pressure and about 9000 for the structure. We have also set $\boldsymbol{c}=\mathbf{0}$ and $\theta(t)=0.2(1-\cos (50 \pi t))$.

In Fig. 6 we show the convergence history of two selected inexact schemes, namely I-GC1-P $\infty$ and I-GC2-P $\infty$, chosen as the most representative, for three selected temporal schemes, namely BDF1/BDF1, BDF2/BDF2 and BDF3/BDF3. A relative $L^{2}$ norm of the error is computed at time $t=0.002 \mathrm{~s}$. The time discretization parameter is $\Delta t=2 \cdot 10^{-3}, 10^{-3}, 5 \cdot 10^{-4}$, 


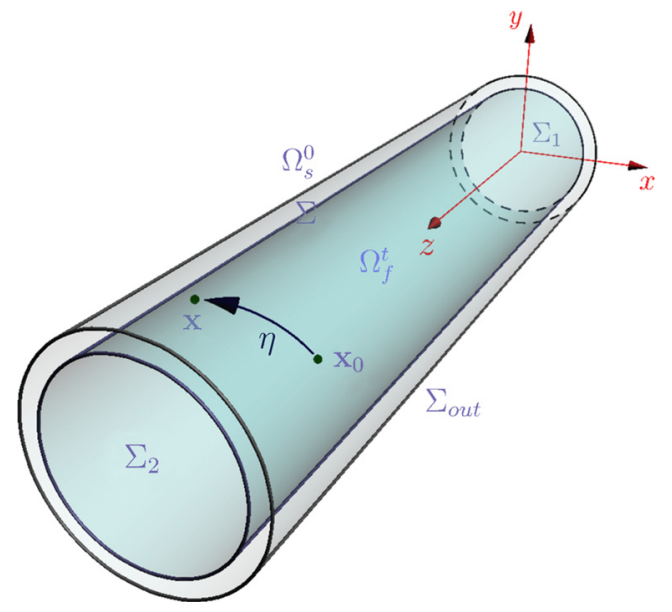

Fig. 5. Cylindrical geometry.
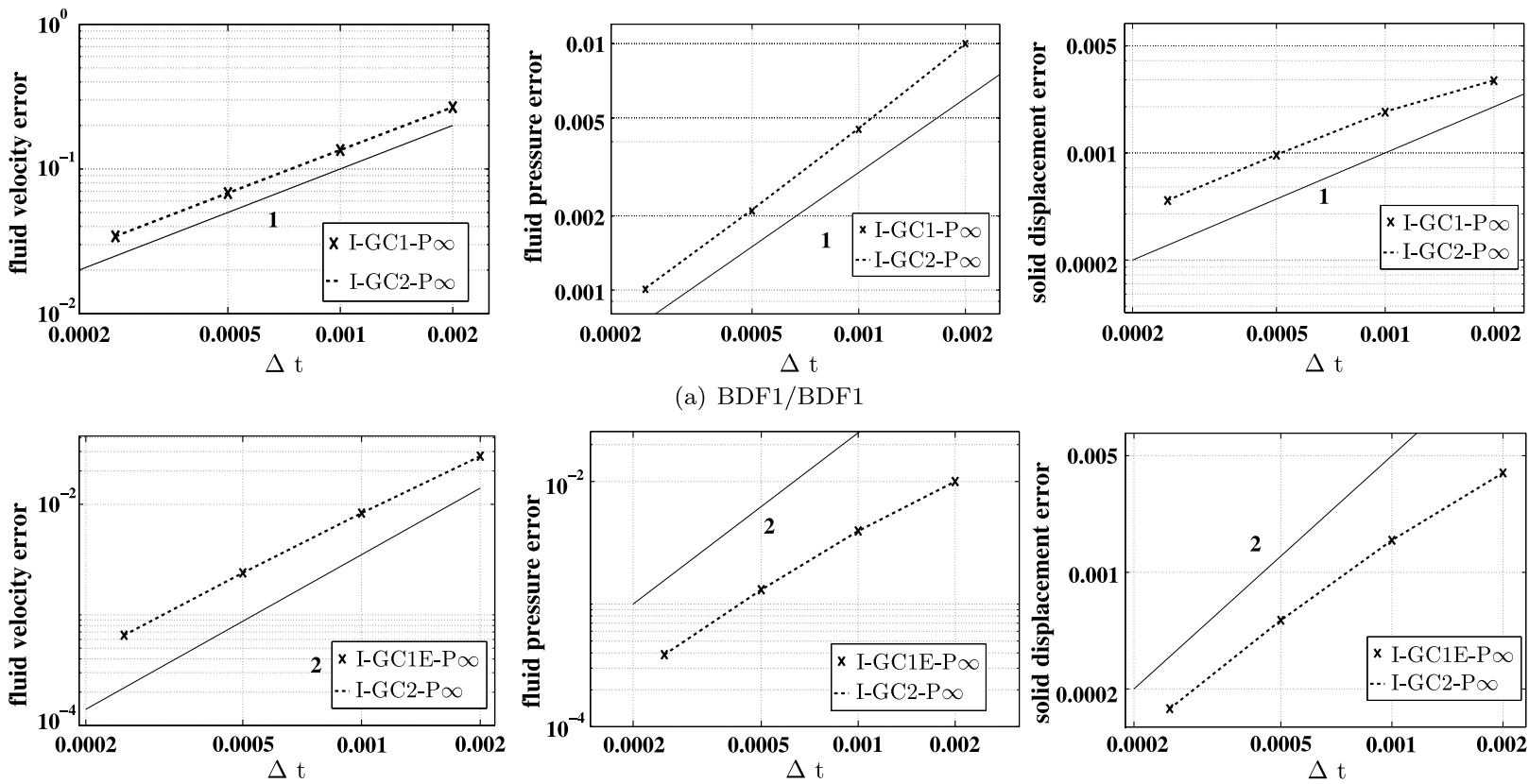

(b) BDF2/BDF2
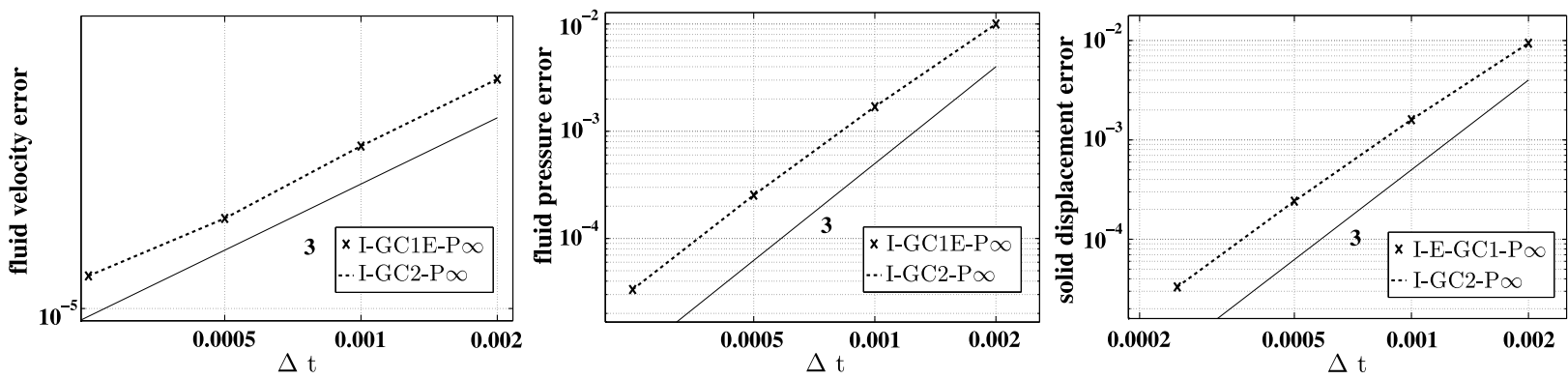

(c) $\mathrm{BDF} 3 / \mathrm{BDF} 3$

Fig. 6. Convergence rate of three temporal schemes considered. Relative errors of the fluid velocity (left), of the pressure (middle) and of the structure displacement (right) - BDF1/BDF1 (a), BDF2/BDF2 (b), BDF3/BDF3 (c) $-t=0.002 \mathrm{~s}$. 
Table 3

Relative error over a selected section of inexact schemes with respect to the exact solution. BDF1/BDF1. Left: displacement. Middle: flow rate. Right: mean pressure. For each box, left: carotid; right: abdominal aorta.

\begin{tabular}{lllllr}
\hline & \multicolumn{3}{c}{$\eta(\%)$} & \multicolumn{3}{c}{$Q(\%)$} \\
\hline I-GC1-P $\infty$ & 0.890 & 0.759 & 0.413 & 0.399 & 0.702 \\
I-GC2-P $\infty$ & 0.003 & 0.002 & 0.005 & 0.004 & 0.003 \\
\hline
\end{tabular}

Table 4

Relative error over a selected section of inexact schemes with respect to the exact solution. BDF2/BDF2. Left: displacement. Middle: flow rate. Right: mean pressure. For each box, left: carotid; right: abdominal aorta.

\begin{tabular}{llllll}
\hline & \multicolumn{3}{c}{$\eta(\%)$} & $Q(\%)$ & \\
\hline I-GC1-P $\infty$ & & & \\
I-GC1E-P $\infty$ & 1.013 & 0.986 & 0.832 & 0.795 & \\
I-GC2-P $\infty$ & 0.066 & 0.059 & 0.084 & 0.081 & 0.187 \\
\end{tabular}

Table 5

Average number of iterations per outer loop in the intermediate and internal ones, and CPU time normalized with respect to that of GC $\infty$-P $\infty$-DT scheme. Inexact schemes. BDF2/BDF2. For each box, left: carotid; right: abdominal aorta.

\begin{tabular}{llllll}
\hline & $\begin{array}{l}\text { \# of external } \\
\text { iterations }\end{array}$ & \multicolumn{2}{l}{$\begin{array}{l}\text { Normalized of internal } \\
\text { CPU time }\end{array}$} & \multicolumn{2}{c}{ iterations } \\
\hline I-GC1-P $\infty$ & 1.0 & 1.0 & 24.8 & 22.8 & 0.34 \\
I-GC1E-P $\infty$ & 1.0 & 1.0 & 24.6 & 22.4 & 0.31 \\
I-GC2-P $\infty$ & 2.0 & 2.0 & 20.3 & 0.33 & 0.29 \\
\hline
\end{tabular}

$2.5 \cdot 10^{-4}$ s. For BDF2/BDF2 and BDF3/BDF3 schemes, I-GC1-P $\infty$ featured just first order convergence, so that we have considered in these cases also the extrapolated versions I-GC1E-P $\infty$.

Discussion of the numerical results. From the convergence rates depicted in Fig. 6, we observe that when BDF1/BDF1 is used, both the inexact schemes recovered first order convergence when a first order extrapolation of the interface position, of the fluid velocity and of the structure displacement is considered. Regarding BDF2/BDF2 and BDF3/BDF3, we observe that I-GC2-P $\infty$ with a first order extrapolation featured second and third order convergence, respectively, whereas for I-GC1E-P $\infty$ an extrapolation of order two and three, respectively, is needed to recover the right convergence order.

These results confirmed the theoretical results found in the previous section (see Table 1, right). In particular, the estimate for the BDF3 method used in combination with 2 approximate-Newton iterations and with a first order extrapolation seems to be not sharp, since from the numerical simulations a third order convergence has been recovered, whereas the theoretical results predicted only a second order convergence.

\subsection{Numerical results for inexact schemes: efficiency and accuracy for two real test cases}

In this section we reported the numerical results obtained for the same test cases presented in Section 5.1 and by using the inexact schemes I-GC1E-P $\infty$ and I-GC2-P $\infty$. This allowed to study the accuracy and the efficiency of such schemes in real contexts.

In Tables 3 and 4 we report the relative errors of the inexact schemes for BDF1/BDF1 and BDF2/BDF2, respectively, by using the solution obtained with GC $\infty-\mathrm{P} \infty$ and the same temporal scheme as the reference one. In particular, we report the $L^{\infty}\left(L^{\infty}\right)$-norm of average quantities, namely the mean structure displacement $\eta$, the flow rate $Q$ and the mean pressure $P$ over a selected section. In Table 5 we report the number of iterations for BDF2/BDF2. In particular, the number of iterations in the internal loops has to be interpreted as the average per outer loop. We also report the CPU time normalized over that of $\mathrm{GC} \infty$-P $\infty$-DT scheme, which is the fastest among the exact schemes.

Discussion of the numerical results. Again, the qualitative trends featured by the numerical performance of the two real cases are in optimal agreement. In particular, the results reported in Tables 3 and 4 show that the relative errors of inexact schemes with respect to the solution obtained with an exact scheme are in any case less than $1.35 \%$. When performing only one external iteration, the accuracy improves of one order of magnitude by performing a second order extrapolation in the case of BDF2/BDF2 (see Table 4). Moreover, when performing two external iterations instead of one the accuracy improves of two orders of magnitude both for BDF1/BDF1 and for BDF2/BDF2. These results suggested that in real applications, an inexact treatment of the structure constitutive non-linearity (in addition to the one related to the geometrical interface condition and to the fluid non-linearity) could be sufficient to recover an accurate solution.

Regarding the efficiency, we found that performing just one external iteration allows a big saving in the computational effort with respect to the exact schemes, the CPU times being reduced up to three times, see Table 5. We did not experience significant differences between the cases with or without extrapolation. When moving from one to two external iterations, the CPU time doubles, being however smaller than the one of the most efficient exact scheme. 


\section{Conclusions}

In this work we studied the numerical performance of some partitioned schemes for the solution of the FSI problem with non-linear fluid and structure subproblems in two real haemodynamic applications. We considered approximate-Newtonbased and classical methods. For the first family of schemes, we considered both exact and inexact schemes, the latter being obtained by performing just one or two iterations in the external loop managing the geometrical coupling and the constitutive non-linearities, guaranteeing in any case the satisfaction of the physical interface conditions. We also performed an error analysis for a model problem.

The main features of such schemes are summarized in what follows:

1. Among exact schemes, approximate-Newton methods are in general better performing than classical schemes which are more than three times slower. In particular, GC $\infty-\mathrm{P} \infty$ seems to be the most efficient for real applications;

2. For the latter scheme, we also experienced the excellent performance of a variant where the physical interface conditions in the internal loop are solved with a precision which is proportional to the external residual (GC $\infty$-P $\infty$-DT). This scheme allowed to almost half the CPU times with respect to $\mathrm{GC} \infty-\mathrm{P} \infty$;

3. Our analysis for a model problem highlighted that performing only $J$ iterations in the approximate-Newton method to solve the non-linear problem arising after the time discretization allows to recover at least order $J$ in time when used in combination with the BDFJ method;

4. Inexact schemes, where both the geometrical interface condition and the constitutive fluid and structure non-linearities are not satisfied exactly, are accurate, recovering, for an analytical 3D test case, the expected convergence rate;

5. Inexact schemes, where two iterations in the external loops are performed, have been seen to be very accurate also for real haemodynamic applications. A very good accuracy has been experienced also when performing just one external iteration for BDF2/BDF2 (even if worse than that obtained with two external iterations), provided that a second order extrapolation is considered. These facts confirm the effectiveness of inexact schemes in haemodynamics, where the structure non-linear problem is not solved exactly;

6. Inexact schemes are more efficient than exact schemes, the CPU time being reduced by a factor three when just one external iteration is performed.

For these reasons, among all the schemes proposed in this work, we recommend I-GC2-P $\infty$ as the best compromise between good accuracy and efficiency for real haemodynamic applications. For BDF2/BDF2 an effective alternative is provided by I-GC1E-P $\infty$, which allows to half the CPU times with respect to I-GC2-P $\infty$, with a slight reduction in the accuracy (the errors being however less than $0.1 \%$ ).

Finally, we stress that the proposed inexact schemes could in principle be applied to any fluid-structure interaction problem. We expect however the accuracy of such schemes to deteriorate for problems characterized by large displacements where the structure non-linearity becomes crucial and should be solved exactly.

\section{Acknowledgements}

This work has been partially supported by the ERC Advanced Grant N.227058 MATHCARD and by the Italian MIUR PRIN09 project n. 2009Y4RC3B_001. The numerical simulations have been performed at CILEA Consortium through a LISA Initiative (Laboratory for Interdisciplinary Advanced Simulation) 2012 grant [link:http://lisa.cilea.it]. The authors would like to thank M. Domanin, L. Forzenigo and P. Biondetti, who have provided the patient images, and L. Azzimonti who have reconstructed the patient-specific flow waveform depicted in Fig. 3, left, from Eco-color Doppler analysis.

\section{References}

[1] M. Astorino, F. Chouly, M. Fernández, Robin based semi-implicit coupling in fluid-structure interaction: stability analysis and numerics, SIAM J. Sci. Comput. 31 (2009) 4041-4065.

[2] M. Astorino, C. Grandmont, Convergence analysis of a projection semi-implicit coupling scheme for fluid-structure interaction problems, Numer. Math. (2010) 721-767.

[3] S. Badia, F. Nobile, C. Vergara, Fluid-structure partitioned procedures based on Robin transmission conditions, J. Comput. Phys. 227 (2008) 7027-7051.

[4] S. Badia, F. Nobile, C. Vergara, Robin-Robin preconditioned Krylov methods for fluid-structure interaction problems, Comput. Methods Appl. Mech. Eng. 198 (2009) 2768-2784.

[5] S. Badia, A. Quaini, A. Quarteroni, Modular vs. non-modular preconditioners for fluid-structure systems with large added-mass effect, Comput. Methods Appl. Mech. Eng. 197 (2008) 4216-4232.

[6] S. Badia, A. Quaini, A. Quarteroni, Splitting methods based on algebraic factorization for fluid-structure interaction, SIAM J. Sci. Comput. 30 (2008) $1778-1805$.

[7] Y. Bazilevs, V. Calo, Y. Zhang, T. Hughes, Isogeometric fluid-structure interaction analysis with applications to arterial blood flow, Comput. Mech. 38 (2006) 310-322.

[8] E. Burman, M.A. Fernández, Explicit strategies for incompressible fluid-structure interaction problems: Nitsche type mortaring versus Robin-Robin coupling, Int. J. Numer. Methods Eng. 97 (10) (2014) 739-758.

[9] P. Causin, J. Gerbeau, F. Nobile, Added-mass effect in the design of partitioned algorithms for fluid-structure problems, Comput. Methods Appl. Mech. Eng. 194 (42-44) (2005) 4506-4527.

[10] P. Crosetto, S. Deparis, G. Fourestey, A. Quarteroni, Parallel algorithms for fluid-structure interaction problems in haemodynamics, SIAM J. Sci. Comput. 33 (4) (2011) 1598-1622. 
[11] J. Degroote, K. Bathe, J. Vierendeels, Performance of a new partitioned procedure versus a monolithic procedure in fluid-structure interaction, Comput. Struct. 11-12 (2009) 793-801.

[12] S. Deparis, M. Fernández, L. Formaggia, Acceleration of a fixed point algorithm for fluid-structure interaction using transpiration conditions, Math. Model. Numer. Anal. 37 (4) (2003) 601-616.

[13] W. Dettmer, D. Perić, On the coupling between fluid flow and mesh motion in the modelling of fluid-structure interaction, Comput. Mech. 43 (2008) 81-90.

[14] J. Donea, An arbitrary Lagrangian-Eulerian finite element method for transient dynamic fluid-structure interaction, Comput. Methods Appl. Mech. Eng. 33 (1982) 689-723.

[15] M. Fernández, J. Gerbeau, C. Grandmont, A projection semi-implicit scheme for the coupling of an elastic structure with an incompressible fluid, Int. J. Numer. Methods Eng. 69 (2007) 794-821.

[16] M. Fernández, M. Moubachir, A Newton method using exact Jacobians for solving fluid-structure coupling, Comput. Struct. 83 (2-3) (2005) 127-142.

[17] M. Fernández, J. Mullaert, M. Vidrascu, Explicit Robin-Neumann schemes for the coupling of incompressible fluids with thin-walled structures, Comput. Methods Appl. Mech. Eng. 267 (2013) 566-593.

[18] C. Figueroa, I. Vignon-Clementel, K. Jansen, T. Hughes, C. Taylor, A coupled momentum method for modeling blood flow in three-dimensional deformable arteries, Comput. Methods Appl. Mech. Eng. 195 (2006) 5685-5706.

[19] L. Formaggia, J.F. Gerbeau, F. Nobile, A. Quarteroni, Numerical treatment of defective boundary conditions for the Navier-Stokes equation, SIAM J. Numer. Anal. 40 (1) (2002) 376-401.

[20] L. Formaggia, A. Quarteroni, A. Veneziani (Eds.), Cardiovascular Mathematics - Modeling and Simulation of the Circulatory System, Springer, 2009.

[21] L. Formaggia, A. Quarteroni, C. Vergara, On the physical consistency between three-dimensional and one-dimensional models in haemodynamics, J. Comput. Phys. 244 (2013) 97-112.

[22] L. Formaggia, A. Veneziani, C. Vergara, Flow rate boundary problems for an incompressible fluid in deformable domains: formulations and solution methods, Comput. Methods Appl. Mech. Eng. 199 (9-12) (2009) 677-688.

[23] L. Formaggia, C. Vergara, Prescription of general defective boundary conditions in fluid-dynamics, Milan J. Math. 80 (2) (2012) 333-350.

[24] C. Forster, W. Wall, E. Ramm, Artificial added mass instabilities in sequential staggered coupling of nonlinear structures and incompressible viscous flow, Comput. Methods Appl. Mech. Eng. 196 (2007) 1278-1293.

[25] M. Gee, U. Kuttler, W. Wall, Truly monolithic algebraic multigrid for fluid-structure interaction, Int. J. Numer. Methods Eng. 85 (8) (2011) $987-1016$.

[26] L. Gerardo Giorda, F. Nobile, C. Vergara, Analysis and optimization of Robin-Robin partitioned procedures in fluid-structure interaction problems, SIAM J. Numer. Anal. 48 (6) (2010) 2091-2116.

[27] M. Heil, An efficient solver for the fully coupled solution of large-displacement fluid-structure interaction problems, Comput. Methods Appl. Mech. Eng. 193 (2004) 1-23.

[28] M. Heil, A. Hazel, J. Boyle, Solvers for large-displacement fluid-structure interaction problems: segregated versus monolithic approaches, Comput. Mech. 43 (1) (2008) 91-101.

[29] T.J.R. Hughes, W.K. Liu, T.K. Zimmermann, Lagrangian-Eulerian finite element formulation for incompressible viscous flows, Comput. Methods Appl. Mech. Eng. 29 (1981) 329-349.

[30] C. Kassiotis, A. Ibrahimbegovic, R. Niekamp, H. Matthies, Nonlinear fluid-structure interaction problem. Part I: implicit partitioned algorithm, nonlinear stability proof and validation examples, Comput. Mech. 47 (2011) 305-323.

[31] C. Kelley, Iterative Methods for Linear and Non-Linear Equations, SIAM, 1995.

[32] R. Kirby, Z. Yosibash, G. Karniadakis, Towards stable coupling methods for high-order discretization of fluid-structure interaction: algorithms and observations, J. Comput. Phys. 223 (2007) 489-518.

[33] U. Kuttler, M. Gee, C. Forster, A. Comerford, W. Wall, Coupling strategies for biomedical fluid-structure interaction problems, Int. J. Numer. Methods Biomed. Eng. 26 (2010) 305-321.

[34] U. Kuttler, W. Wall, Fixed-point fluid-structure interaction solvers with dynamic relaxation, Comput. Mech. 43 (2008) 61-72.

[35] Y. Liu, C. Charles, M. Gracia, H. Gregersen, G.S. Kassab, Surrounding tissues affect the passive mechanics of the vessel wall: theory and experiment, Am. J. Physiol., Heart Circ. Physiol. 293 (2007) H3290-H3300.

[36] H. Matthies, R. Niekamp, J. Steindorf, Algorithms for strong coupling procedures, Comput. Struct. 195 (2006) 2028-2049.

[37] G. Mengaldo, P. Tricerri, P. Crosetto, S. Deparis, F. Nobile, L. Formaggia, A comparative study of different nonlinear hyperelastic isotropic arterial wall models in patient-specific vascular flow simulations in the aortic arch, MOX-Report n. 15-2012, Department of Mathematics, Politecnico di Milano, Italy, 2012.

[38] P. Moireau, N. Xiao, M. Astorino, C.A. Figueroa, D. Chapelle, C.A. Taylor, J.F. Gerbeau, External tissue support and fluid-structure simulation in blood flows, Biomech. Model. Mechanobiol. 11 (1-2) (2012) 1-18.

[39] C.M. Murea, S. Sy, A fast method for solving fluid-structure interaction problems numerically, Int. J. Numer. Methods Fluids 60 (2009) $1149-1172$.

[40] F. Nobile, Numerical approximation of fluid-structure interaction problems with application to haemodynamics, Ph.D. thesis, École Polytechnique Fédérale de Lausanne, 2001, Thesis nº 2458.

[41] F. Nobile, M. Pozzoli, C. Vergara, Time accurate partitioned algorithms for the solution of fluid-structure interaction problems in haemodynamics, Comput. Fluids 86 (2013) 470-482.

[42] F. Nobile, C. Vergara, An effective fluid-structure interaction formulation for vascular dynamics by generalized Robin conditions, SIAM J. Sci. Comput. 30 (2008) 731-763.

[43] F. Nobile, C. Vergara, Partitioned algorithms for fluid-structure interaction problems in haemodynamics, Milan J. Math. 80 (2012) $443-467$.

[44] K. Perktold, E. Thurner, T. Kenner, Flow and stress characteristics in rigid walled and compliant carotid artery bifurcation models, Med. Biol. Eng. Comput. 32 (1994) 19-26.

[45] A. Quarteroni, R. Sacco, F. Saleri, Numerical Mathematics, Springer, Berlin, 2000.

[46] E. Swim, P. Seshaiyer, A nonconforming finite element method for fluid-structure interaction problems, Comput. Methods Appl. Mech. Eng. (2006) 2088-2099.

[47] T. Tezduyar, S. Sathe, T. Cragin, B. Nanna, B. Conklin, J. Pausewang, M. Schwaab, Modelling of fluid-structure interactions with the space-time finite elements: arterial fluid mechanics, Int. J. Numer. Methods Fluids 54 (2007) 901-922.

[48] T. Tezduyar, S. Sathe, K. Stein, Solution techniques for the fully discretized equations in computation of fluid-structure interactions with the space-time formulations, Comput. Methods Appl. Mech. Eng. 195 (41-43) (2006) 5743-5753.

[49] A. Veneziani, C. Vergara, Flow rate defective boundary conditions in haemodynamics simulations, Int. J. Numer. Methods Fluids 47 (2005) $803-816$.

[50] C. Vergara, Nitsche's method for defective boundary value problems in incompressibile fluid-dynamics, J. Sci. Comput. 46 (1) (2011) $100-123$.

[51] Y. Yu, H. Baek, G. Karniadakis, Generalized fictitious methods for fluid-structure interactions: analysis and simulations, J. Comput. Phys. 245 (2013) $317-346$. 